POSITION PAPER

\title{
A framework for the development of effective anti-metastatic agents
}

\author{
Robin L. Anderson ${ }^{1,2,3}$, Theo Balasas ${ }^{4}$, Juliana Callaghan ${ }^{5}$, R. Charles Coombes ${ }^{6}$, \\ Jeff Evans ${ }^{7}$, Jacqueline A. Hall, Sally Kinrade ${ }^{3,9}$, David Jones ${ }^{10}$, Paul S. Jones ${ }^{11}$, \\ Rob Jones ${ }^{7}$, John F. Marshall'2, Maria Beatrice Panico ${ }^{10}$, Jacqui A. Shaw ${ }^{13}$, \\ Patricia S. Steeg ${ }^{14}$, Mark Sullivan ${ }^{3,9}$, Warwick Tong ${ }^{3}$, Andrew D. Westwell ${ }^{15}$ and \\ James W. A. Ritchie ${ }^{4,11 *}$, on behalf of the Cancer Research UK and Cancer Therapeutics \\ CRC Australia Metastasis Working Group
}

Abstract | Most cancer-related deaths are a result of metastasis, and thus the importance of this process as a target of therapy cannot be understated. By asking "how can we effectively treat cancer?', we do not capture the complexity of a disease encompassing > 200 different cancer types - many consisting of multiple subtypes - with considerable intratumoural heterogeneity, which can result in variable responses to a specific therapy. Moreover, we have much less information on the pathophysiological characteristics of metastases than is available for the primary tumour. Most disseminated tumour cells that arrive in distant tissues, surrounded by unfamiliar cells and a foreign microenvironment, are likely to die; however, those that survive can generate metastatic tumours with a markedly different biology from that of the primary tumour. To treat metastasis effectively, we must inhibit fundamental metastatic processes and develop specific preclinical and clinical strategies that do not rely on primary tumour responses. To address this crucial issue, Cancer Research UK and Cancer Therapeutics CRC Australia formed a Metastasis Working Group with representatives from not-for-profit, academic, government, industry and regulatory bodies in order to develop recommendations on how to tackle the challenges associated with treating (micro)metastatic disease. Herein, we describe the challenges identified as well as the proposed approaches for discovering and developing anticancer agents designed specifically to prevent or delay the metastatic outgrowth of cancer.

*e-mail: james.ritchie@ cancer.org.uk https://doi.org/10.1038/ s41571-018-0134-8
Metastasis of cancer to distal sites is associated with poor patient prognosis and is the foremost cause of cancer-related death ${ }^{1}$, with approximately $90 \%$ of patients who succumb to cancer dying of metastatic disease $^{2}$. Despite the advent of effective immunotherapies within the past decade, the majority of patients with advanced-stage and/or high-risk cancers continue to die as a direct result of metastatic disease or owing to complications of its treatment ${ }^{3}$. Indeed, improvements in the survival of patients with cancer over time have not equally benefited those with metastatic disease ${ }^{4}$.

Nevertheless, most standard-of-care treatments and new molecularly targeted therapies, including immunotherapies, were developed on the basis of initial evidence of anticancer activity - either direct or via immune system engagement - obtained in preclinical studies with tumorigenesis and/or primary growth, not metastatic activity, as the main end points. Similarly, clinical drug development generally relies on the demonstration of tumour shrinkage according to the radiological Response Evaluation Criteria for Solid Tumors (RECIST) ${ }^{5,6}$, with confirmatory improvements in clinical outcomes, ignoring the ability to inhibit metastasis. Only after clinically meaningful tumour responses and/or improvements in patient survival have been demonstrated in the metastatic setting will the drug be tested in adjuvant trials, with the aim of preventing or delaying the development of overt metastatic disease. Consequently, a paucity of preclinical discovery and thus clinical development exists for agents targeting the biological mechanisms underlying the metastatic process.

An urgent need remains for novel therapeutic strategies and agents that prevent the establishment of and/or tissue colonization by metastases, which ultimately 


\section{Key points \\ - Metastasis is associated with a poor patient prognosis and is the foremost cause of cancer-related death, with approximately $90 \%$ of patients who succumb to cancer dying of metastatic disease. \\ - Metastasis is inherently complex, with different distant sites having a distinct and specific extracellular matrix and cellular composition compared with that of the originating site, and therefore metastases must be considered biologically different from the primary tumour. \\ - The standard cancer drug discovery and development pathway, including that for molecularly targeted and immunotherapies, generally ignores the ability of experimental medicines to inhibit metastasis. \\ - A wealth of potential preclinical targets for anti-metastatic drug discovery and development have already been identified but remain to be validated using appropriate preclinical models that reflect the pathogenesis of metastatic disease in patients. \\ - Despite some successes in the treatment of bone metastases, following extensive analyses in preclinical models, multiple late-stage failures in clinical development have resulted in anti-metastatic drug development efforts being deprioritized by the pharmaceutical industry. \\ - Successful development of effective anti-metastatic therapies will require the regulatory agencies to work together with researchers, drug developers and statisticians to redefine the clinical development paradigm in order to encourage development of this complex but high-potential category of oncology drugs.}

lead to organ failure, morbidity and death. In addition, new strategies must be developed to facilitate clinical testing of therapeutic agents with an anti-metastatic mechanism of action. To assist the cancer drug discovery and development community in addressing this critical issue, Cancer Research UK (CRUK), Cancer Research Technology (CRT) and Cancer Therapeutics CRC Australia (CTx) formed a Metastasis Working Group with representatives from academia, industry, government and regulatory bodies in order to develop recommendations on how to surmount the challenges associated with treating metastatic disease. This article provides an overview of these challenges and describes the Metastasis Working Group recommendations on best practices for the discovery and development of anticancer agents designed specifically

\section{Author addresses}

${ }^{1}$ Translational Breast Cancer Program, Olivia Newton-John Cancer Research Institute, Heidelberg, Victoria, Australia.

${ }^{2}$ School of Cancer Medicine, La Trobe University, Bundoora, Victoria, Australia.

${ }^{3}$ Cancer Therapeutics Cooperative Research Centre (CTx), Melbourne, Victoria,

Australia.

${ }^{4}$ Commercial Partnerships, Cancer Research UK (CRUK), London, UK.

${ }^{5}$ Research and Innovation Services, University of Portsmouth, Portsmouth,

Hampshire, UK.

${ }^{6}$ Department of Surgery and Cancer, Imperial College London, Hammersmith Hospital,

London, UK.

${ }^{7}$ Institute of Cancer Sciences, University of Glasgow, Glasgow, Scotland, UK.

${ }^{8}$ Research and Development, Vivacitv Ltd, Chesham, Buckinghamshire, UK.

${ }^{9}$ Medicines Development for Global Health, Southbank, Victoria, Australia.

${ }^{10}$ Medicines and Healthcare Products Regulatory Agency, London, UK.

${ }^{11}$ Centre for Drug Development, CRUK, London, UK.

${ }^{12}$ Queen Mary University of London, Barts Cancer Institute, London, UK.

${ }^{13}$ Leicester Cancer Research Centre, University of Leicester, Leicester, Leicestershire, UK.

${ }^{14}$ Women's Malignancies Branch, Center for Cancer Research, National Cancer Institute,

Bethesda, MD, USA.

${ }^{15}$ School of Pharmacy and Pharmaceutical Sciences, Cardiff University, Cardiff, Wales, UK.
}

to circumvent metastasis, with consideration given to their implementation in clinical trials.

\section{Challenges}

The development of new effective medicines that interrupt the primary causes of metastasis is a daunting but important challenge. Mechanistically, metastatic tumour cells are genetically unstable, and in most cancers no single dominant pathway is likely to control metastasis? Indeed, the signalling pathways driving metastasis can vary between primary and secondary tumours and between metastases that arise at different sites ${ }^{8}$. The target of translational research efforts is often an occult population of tumour cells disseminating from the primary tumour, sitting dormant in a sanctuary site or constituting a micrometastasis in a distant organ. Selection for clinical testing of candidate anti-metastatic agents that might have limited or no effect on conventional preclinical outcomes, such as primary tumour growth, is another key challenge. In addition, validated biomarkers that can be used to increase the efficiency of mechanistic experiments and accelerate drug development are rarely available.

Across all cancers, the extent to which tumour cells have left the primary tumour and established occult (micro)metastases before patient diagnosis is acknowledged to be poorly characterized ${ }^{9,10}$. Tumour cells might become invasive early in cancer development, and thus prevention of dissemination from a primary tumour is unlikely to be a clinically successful strategy owing to the presence of pre-existing but undetected metastases; however, prevention of secondary metastases is a plausible rationale for intervention. Metastatic dissemination is a multistage process, and various points of intervention have been identified and credentialed at the preclinical level (FIG. 1). These include targeting the initial steps of invasion and migration away from the primary tumour, entry into the circulation (intravasation) and extravasation at a distant site. As with invasion, traversal of the circulatory system might also be an early event in cancer development, leading to the proposal that drug development efforts should also take into consideration the abrogation of metastatic colonization - that is, the outgrowth of a lesion in a foreign environment. Several aspects of metastatic colonization are distinct from primary tumour formation and could influence drug development. Moreover, multiple studies have revealed that tumour cells colonizing distant organs can differ from those of the primary tumour in many respects $\mathrm{s}^{10-13}$, revealing potential therapeutic targets. Changes in tissue microenvironments that facilitate metastatic colonization are incompletely characterized and might begin before the arrival of metastatic tumour cells ${ }^{14,15}$. Several approaches for targeting such secondary sites have been proposed. Intervening early to disrupt the 'pre-metastatic niche' is one potential strategy, while therapy to either maintain dormancy or induce the death of cells in micrometastatic lesions is another. Whereas metastases that are detected before commencement of first-line therapy can sometimes be treated using radiation therapy, new and less deleterious therapies that are additive or synergistic with the standards of care are a priority for the treatment of occult metastatic disease. 


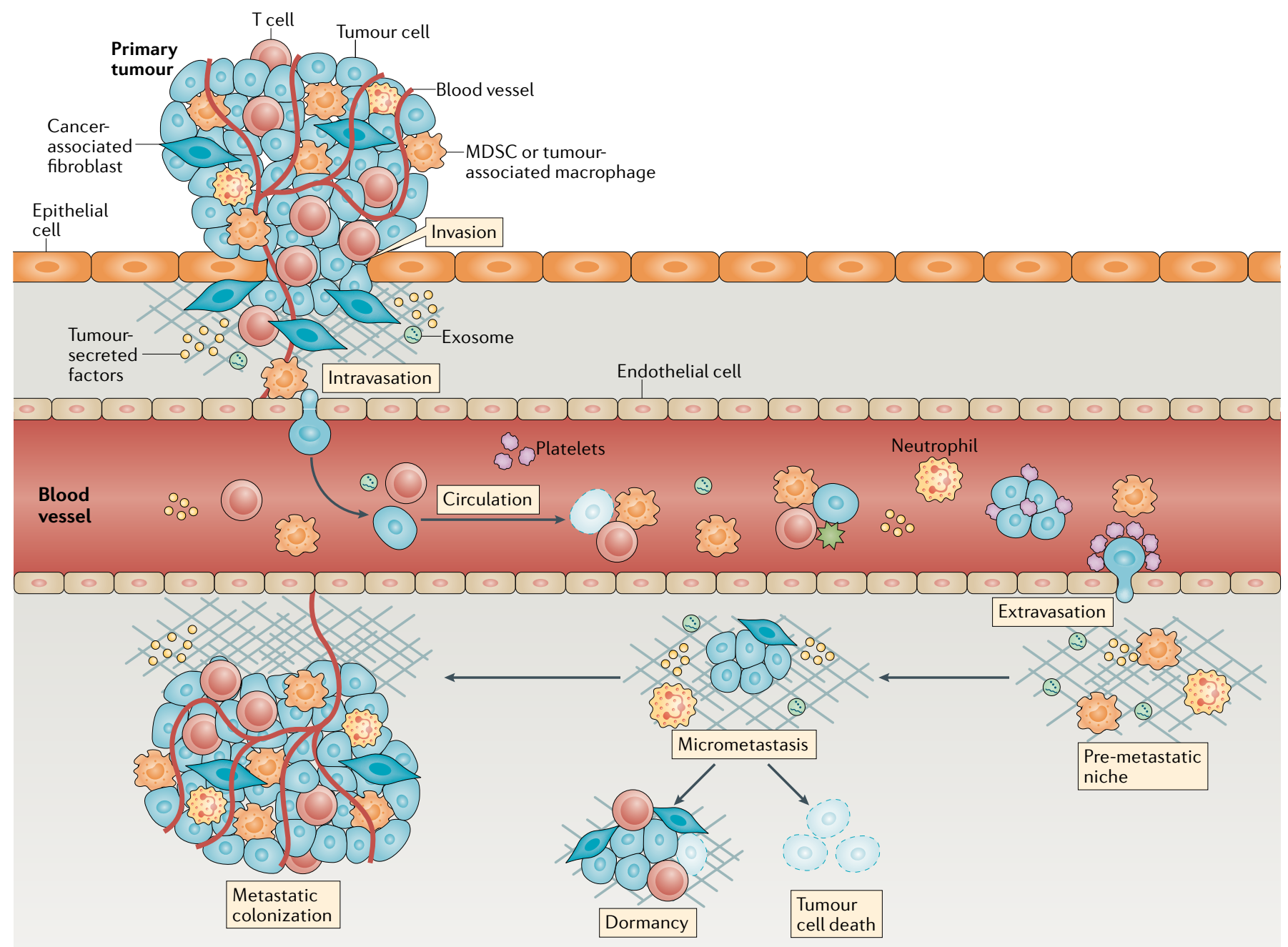

Fig. 1 Overview of metastasis. Metastasis is a complex multistep process, and the very concept of designing a metastasisspecific therapeutic must consider which part of the process is best to target. Given that metastases are derived mainly from invasive tumours, therapeutic efforts have often targeted the intrinsic invasive propensity of tumour cells ${ }^{150,151}$. Tumour cell production of angiogenic factors and TGF $\beta$ can activate endothelial cells and fibroblasts to remodel tissues and promote tumour cell invasion of stromal-modified spaces ${ }^{152}$. Targeting stromal elements in cancers remains an active area of research ${ }^{153-157}$. Intravasation of tumour cells is promoted by binding to macrophages that cause transient permeability in the vasculature ${ }^{158}$; thus, targeting tumour-associated macrophages might reduce the number of circulating tumour cells $(\mathrm{CTCs})^{159}$. Multiple factors intrinsic to tumour cells (including epithelial-to-mesenchymal transition, production of proteases and migratory capacity) improve intravasation, often via effects on cell types including fibroblasts, neutrophils and macrophages ${ }^{160}$. Most tumour cells that enter the vasculature die as a result of hydrodynamic physical damage or leukocyte attack $^{160}$. However, platelets can bind to and protect CTCs and improve their ability to establish secondary sites ${ }^{161}$. PlateletCTC aggregates settled at distant sites can release cytokines that attract granulocytes ${ }^{162}$; targeting platelets or granulocyte recruitment can prevent metastasi ${ }^{162}$. Additionally, abrogation of platelet-CTC binding, leading to a reduction in the number of circulating and potentially metastatic cells ${ }^{163}$, might explain the suppression of metastasis by aspirin in breast and prostate cancer model ${ }^{164}$. Survival and proliferation of newly deposited cancer cells in a metastatic site are arguably the most important stages of the metastatic process. Cancers with a propensity to metastasize do not grow in all organs, indicating that a limited number of organs provide a suitable stromal environment for their colonization. Preferred colonization sites, termed pre-metastatic niches, can be prepared in advance of the arrival of disseminated tumour cells through the actions of myeloid-derived suppressor cells (MDSCs) and tumour cell-derived extracellular vesicles (EVs), such as exosomes ${ }^{17,165}$. Whether this process can provide novel therapeutic targets to limit the arrest and survival of metastatic cells remains unclear, with development of EV-specific drugs, for example, creating a challenge ${ }^{166}$. Evidence also supports roles for neutrophils ${ }^{167,168}$ and MDSCs ${ }^{169}$ in metastatic colonization. Evasion of the antitumour immune response is another critical factor in metastatic colonization. No single tumour type seems to exhibit all these mechanisms; therefore, targeting any one stage of the metastatic process requires a tumour-specific understanding of the mechanisms involved.

The metastatic cell is a single genetic entity derived from a mass of cells possessing extensive genetic heterogeneity and consequent plasticity ${ }^{8}$. The distant tissue site will also have a distinct and specific extracellular matrix and cellular composition compared with that of the tumour tissue from which the metastatic cell originated. Metastases must therefore be considered biologically different from primary tumour cells, at least in the early stages of 
outgrowth. Studies specifically designed to examine the biological development of metastases are not common, and if we hope to target metastases effectively, we must gain a more complete understanding of the underlying biology. This issue is complex, although some successes have already been achieved, for example, in understanding the processes of pancreatic cancer metastasis ${ }^{16,17}$.

Metastatic dormancy is defined by an unusually long disease-free interval (months, years or even decades depending on the cancer type) between removal or successful therapy of the primary tumour and subsequent clinical relapse with disseminated disease. Metastatic dormancy can be achieved by many means. Tumour cells can exit from the cell cycle or balance their proliferation and apoptosis. Host cells can limit angiogenesis or alter anticancer immune responses, resulting in immunoediting (with an equilibrium between immune elimination and escape of tumour cells) ${ }^{18}$. Tumour cell dormancy also alters chemotherapeutic efficacy, either because the non-dividing cells are more resistant to such treatment ${ }^{19}$ or because they are protected by cellular and extracellular components of their microenvironment ${ }^{20-22}$ (FIG. 2).

The optimal means of selecting patients with a predictable risk and rate of disease progression for enrolment in a clinical trial of an anti-metastatic agent remain largely unknown. This challenge is compounded by the inability to reliably quantify the prevalence and extent of occult metastatic disease at enrolment. Because adjuvant clinical trials are often conducted only after positive results have been obtained in early phase clinical studies involving patients with advanced-stage (metastatic) disease and require considerable funding, large numbers of patients and long follow-up durations to capture the primary outcome measure (for example, disease-free survival (DFS)), new non-conventional clinical development approaches are needed. The clinical trial design might vary depending

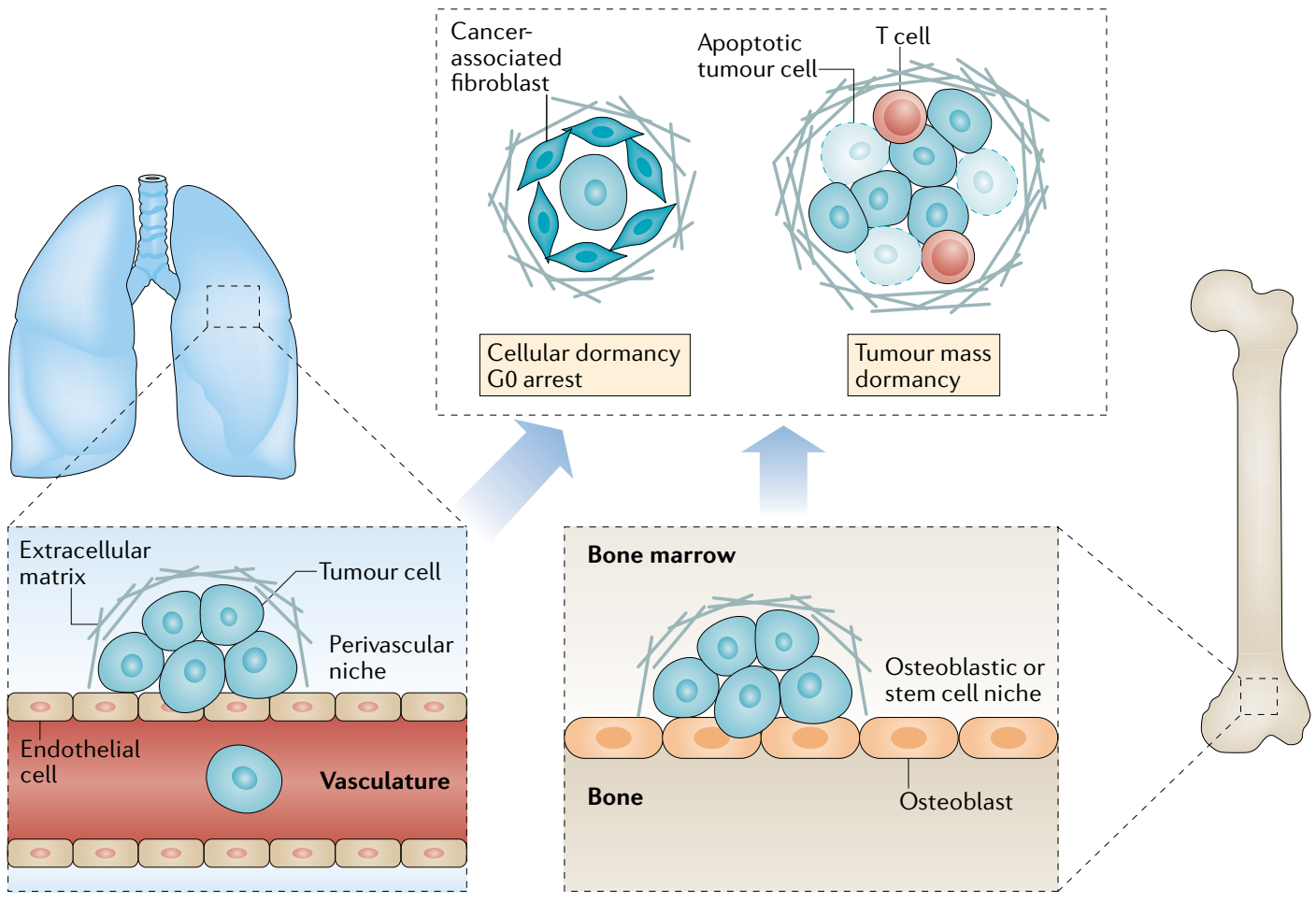

Fig. 2 | Dormancy and the metastatic niche. Metastatic latency is more pronounced for certain types of cancer, notably breast and prostate cancer and melanoma ${ }^{170}$. The detection of disseminated tumour cells (DTCs) in bone marrow aspirates, obtained long after eradication of the primary tumour, verifies the presence of dormant tumour cells and is predictive of disease recurrence ${ }^{171-173}$. Dormant tumour cells are proposed to exist either as single cells in a state of cell cycle arrest or as small masses of cells that fail to expand into clinically detectable lesions, possibly owing to failure of angiogenesis, balanced rates of proliferation and apoptosis or effective immunosurveillance ${ }^{174}$. Cells derived from these clusters that are too small to be detected by normal clinical imaging are presumably the source of circulating tumour cells detected in some patients after successful treatment of the primary tumour. The metastatic niche is likely to vary between different organs, reflecting the tissue-specific nature of the microenvironment in which the DTC is located; extensive crosstalk occurs between the tumour cells, stromal cells and extracellular matrix components of the niche. Various niches have been proposed, including the perivascular niche associated with the vasculature ${ }^{22}$, the haematopoietic stem cell niche of the bone marrow ${ }^{175}$ and the osteoblastic niche in bone ${ }^{176}$. The factors that maintain tumour cell dormancy in these niches are starting to be unravelled, with different extracellular matrix components, cytokines and other proteins being implicated for different cancer types and niches. Far less is understood about how dormancy is broken, which could occur following failure of immunosurveillance, in response to inflammation triggered by trauma or an infection or perhaps as a result of ageing-related deficiencies in tissue homeostasis. With regard to therapy, the challenge is to decide whether the aim should be to retain the DTCs in a dormant state or, instead, to disrupt their niche and dormancy, thereby rendering them susceptible to apoptotic or anoikic death and/or to chemotherapy. 
on the patient population, the dosing schema (maximum tolerated versus biologically effective dose, limited cycles versus maintenance therapy and scheduling in relation to standard-of-care therapy), the primary end points and the available biomarkers of activity. In most cases, such trials will also need to be initiated without a history of regulatory approval of the experimental agent.

\section{Missteps and a home run}

Several past efforts to target the mechanisms of tumour metastasis have resulted in failure. Matrix metalloproteinase (MMP) inhibitors were developed as antimetastatic agents on the basis of their capacity to inhibit tissue invasion by tumour cells early in the metastatic cascade and their cytostatic activity in preclinical mod$\mathrm{els}^{23,24}$. Subsequent studies enabled a more nuanced understanding of the metastasis-promoting as well as the metastasis-limiting effects of the MMPs and revealed that several other proteases have overlapping functions ${ }^{25}$. The preclinical models used had limited relevance to the clinical testing scenario, with the experimental design typically involving initiation of MMP inhibitor treatment soon after tumour cell inoculation, to form either a primary tumour or experimental metastases. In this setting, the MMP inhibitors were shown to be effective $^{26}$; however, in the clinic, MMP inhibitors were tested in patients with advanced-stage disease, usually with drug-resistant metastatic disease. As a result, these phase II and phase III clinical trials of MMP inhibitors failed to show strong signals of efficacy ${ }^{27,28}$. In addition, the early drugs had broad spectrum anti-protease activity and were characterized by serious adverse effects in patients ${ }^{26}$. Other examples of anti-metastatic agents for which promising preclinical activity has not been translated into clinical efficacy include cilengitide (targeting $\alpha v \beta 3$ and $\alpha v \beta 5$ integrins on angiogenic blood vessels) ${ }^{29}$, and dasatinib and saracatinib (targeting SRC and BCR$\mathrm{ABL} 1)^{4}$. These late-stage failures in clinical development have resulted in major financial losses, leading to antimetastatic drug development being deprioritized by the pharmaceutical industry.

Conversely, successes have been achieved in the treatment of bone metastases, following extensive analyses in preclinical models, either with antibodies targeting receptor activator of NF- $\mathrm{\kappa B}$ ligand (RANKL; also known as TNFSF11) or with a class of drugs known as bisphosphonates. Both of these therapies interrupt the 'vicious cycle' of bone metastasis. The bone metastatic vicious cycle is a specific example of tumour cell-microenvironment interactions that are likely to occur at other metastatic sites (albeit via differing mechanisms). In bone, tumour cells produce factors that activate osteoblasts to produce RANKL, which activates osteoclasts that subsequently degrade bone, releasing a host of growth factors that stimulate metastatic colonization by tumour cells ${ }^{30}$. Denosumab is a humanized monoclonal antibody targeting RANKL that has been shown in preclinical experiments to bind with its target in the bones of healthy transgenic mice expressing chimeric mouse-human $\mathrm{RANKL}^{31}$. In initial clinical trials in the metastatic setting, traditional tumour growth inhibition or survival end points were not used as outcome measures with this cytostatic agent; instead, a reduction in skeletalrelated events (SREs) was the primary end point ${ }^{32,33}$. Deleterious SREs, such as a bone fracture from expansion of an existing metastasis or a new metastasis, were essentially direct readouts of the extent of metastatic burden. Accordingly, patients with breast or prostate cancers were enrolled because they are prone to developing bone metastases. Significant reductions in the incidence of SREs in patients treated with denosumab compared with those who received standard care were observed for both types of cancer ${ }^{34,35}$. In men with bone-metastatic castration-resistant prostate cancer (CRPC), the median time to first on-study SRE with denosumab was 20.7 months versus 17.1 months with the bisphosphonate zoledronic acid (HR 0.82, 95\% CI $0.71-0.95 ; P=0.0002$ for non-inferiority; $P=0.008$ for superiority $)^{34}$. In women with advanced-stage breast cancer, denosumab was superior to zoledronic acid in delaying the time to first on-study SRE (HR 0.82, 95\% CI $0.71-0.95 ; P=0.01$ ) and also the time to first and subsequent on-study SREs (rate ratio 0.77, 95\% CI $0.66-0.89 ; P=0.001)^{35}$. Denosumab was then compared with placebo in adjuvant trials and delayed initial bone metastasis in patients with CRPC by a median of 4.2 months $^{36}$. However, in placebo-controlled trials involving patients with breast cancer, adjuvant denosumab was associated with only a minor ${ }^{37}$ or no $^{38}$ reduction in the DFS or overall survival (OS). The reasons for this disparity between SREs and OS outcomes are not entirely clear but might reflect a shift in bone metabolism that reduces the incidence of SREs without direct effects on cancer progression.

The value of bisphosphonates in reducing bone resorption and bone metastasis through direct targeting of osteoclasts has been demonstrated in many preclinical studies ${ }^{39}$. In addition, bisphosphonates have broader anticancer activity against metastatic lesions in visceral organs, possibly through inhibition of angiogenesis or through inhibition of M2-like macrophages in other tissues $^{39,40}$. A meta-analysis of clinical trials using bisphosphonates, supported by earlier preclinical data, has demonstrated reduced bone metastases and prolonged OS, at least in postmenopausal women with early stage breast cancer ${ }^{41}$. No benefit of bisphosphonate treatment was observed for premenopausal women ${ }^{41}$.

\section{Preclinical drug development Target identification}

Many targets with known or proposed roles in metastasis could be candidates for drug development (TABLE 1). These potential targets have been identified on the basis of associations between gene targets (often mutated) and metastasis or poor survival in patients $\mathrm{s}^{42,43}$, targeted manipulation of genes to alter metastasis in preclinical models ${ }^{44-46}$, functional genomic screens ${ }^{47-49}$ and drug repurposing efforts ${ }^{50}$. In addition to functional preclinical experiments, evidence that the target is associated with metastasis in the human disease is required. For the development of a targeted therapy, such as a small molecule, a peptide or an antibody, knowledge of the biological activity of the target is essential for supporting development of a functional biomarker. Targets 
Table 1 | Selected preclinical data for potential anti-metastatic therapies

\begin{tabular}{|c|c|c|}
\hline Agent & Target & Preclinical data $^{a}$ \\
\hline \multicolumn{3}{|l|}{ Antibodies } \\
\hline Anti-CCL2 & CCL2 (chemokine) & $\begin{array}{l}\text { Prevented mobilization of myeloid cells from the bone marrow } \\
\text { to colorectal liver metastases and thereby reduced metastasis }{ }^{177}\end{array}$ \\
\hline Anti-BMP6 & BMP6 (TGF $\beta$ superfamily cytokine) & Reduced osteoblastic bone metastasis from prostate cancer ${ }^{178}$ \\
\hline Anti-PTHrP & PTHrP (hormone involved in bone vicious cycle) & Reduced liver and bone metastasis of melanoma ${ }^{179}$ \\
\hline Anti-N-cadherin & $\mathrm{N}$-cadherin (mesenchymal cadherin) & $\begin{array}{l}\text { Reduced prostate cancer muscle invasion and induced tumour } \\
\text { cell apoptosis }{ }^{180}\end{array}$ \\
\hline Anti-CD24 & CD24 (GPI-linked sialo-glycoprotein) & Reduced lung metastasis of bladder cancer ${ }^{181}$ \\
\hline Anti-CDCP1 & Protease cleavage site of CDCP1 & $\begin{array}{l}\text { Prevented lung metastasis by inducing poly(ADP-ribose) } \\
\text { polymerase-mediated cell death }{ }^{182}\end{array}$ \\
\hline Anti-TSPAN8 & TSPAN8 (tumour-associated tetraspanin) & Reduced metastasis of epithelial ovarian cancer ${ }^{183}$ \\
\hline Anti-MT1-MMP & Membrane type 1 MMP & Reduced lung metastasis of melanoma ${ }^{184}$ \\
\hline \multicolumn{3}{|l|}{ Small-molecule inhibitors } \\
\hline BL5923 & CCR1 (CCL9 and/or CCL15 chemokine signalling) & $\begin{array}{l}\text { Inhibited liver metastasis of CRC by blocking recruitment } \\
\text { of myeloid cells }{ }^{185}\end{array}$ \\
\hline SD208 & TGF $\beta$ receptor 1 & $\begin{array}{l}\text { Reduced melanoma and prostate bone metastasis and } \\
\text { decreased progression of established lesions }{ }^{186,187}\end{array}$ \\
\hline CCT129254 & Multiple kinases (including ROCK, PI3K and AKT) & Inhibited melanoma lung metastasis ${ }^{188}$ \\
\hline Zibotentan & Endothelin 1 & $\begin{array}{l}\text { Prevented lung colonization by bladder cancer cells but had no } \\
\text { effect on established metastases }{ }^{189}\end{array}$ \\
\hline Debio 0719 & Lysophosphatidic acid receptor 1 (fibrosis) & $\begin{array}{l}\text { Decreased lung and liver metastasis in breast cancer and } \\
\text { induced tumour cell dormancy }{ }^{11}\end{array}$ \\
\hline$\beta$-Aminopropionitrile & Lysyl oxidases & $\begin{array}{l}\text { Prevented breast cancer metastasis but had no effect on existing } \\
\text { lesions }^{190}\end{array}$ \\
\hline ССТ365623 & Lysyl oxidases & Prevented metastasis of breast cancer ${ }^{191}$ \\
\hline CA-074 & Cathepsin B inhibitor & $\begin{array}{l}\text { Prevention of bone metastasis and shrinkage of existing bone } \\
\text { metastases in a breast cancer model }{ }^{192}\end{array}$ \\
\hline Napabucasin & Unclear (STAT3 and cancer stem cell pathways) & Reduced metastasis of pancreatic and colon cancers ${ }^{193}$ \\
\hline HO-3867 & $\begin{array}{l}\text { Unclear (STAT3 signalling and reversion of mutant } \\
\text { p53 to a wild-type phenotype) }\end{array}$ & Reduced metastasis of ovarian cancer ${ }^{194}$ \\
\hline $\begin{array}{l}\text { IRAK inhibitor and } \\
\text { ginsenosides }\end{array}$ & IRAK1 & $\begin{array}{l}\text { Reversed paclitaxel resistance and reduced metastasis of } \\
\text { TNBC }{ }^{195}\end{array}$ \\
\hline Bafetinib & LYN and BCR-ABL1 & Decreased liver metastasis in a breast cancer model ${ }^{196}$ \\
\hline KPT-6566 & $\begin{array}{l}\text { PIN1 (prolyl isomerase that regulates proline- } \\
\text { directed kinase signalling) }\end{array}$ & Decreased lung metastasis of TNBC ${ }^{197}$ \\
\hline SF2523 & $\begin{array}{l}\text { Dual PI3K and BRD4 inhibitor (MYC-mediating } \\
\text { factors) }\end{array}$ & $\begin{array}{l}\text { Reduced regional colonic lymph node metastasis and shrank } \\
\text { established metastases in pancreatic carcinoma mode }{ }^{198}\end{array}$ \\
\hline Nifuroxazide & Unclear (STAT3 signalling) & $\begin{array}{l}\text { Inhibited lung and abdomen metastasis of CRC and shrank } \\
\text { existing metastases }{ }^{199}\end{array}$ \\
\hline AECHL-1 (triterpenoid) & $\begin{array}{l}\text { Unclear (alters cytoskeletal dynamics and inhibits } \\
\text { NF-kB-mediated MAPK activity) }\end{array}$ & Decreased lung metastasis of TNBC ${ }^{200}$ \\
\hline CCG-203971 & Unclear (inhibits the RHO-MRTF-SRF pathway) & Decreased lung metastasis in melanoma mode $\mathrm{l}^{201}$ \\
\hline Regorafenib & $\begin{array}{l}\text { Multiple kinases (including angiogenic receptor } \\
\text { tyrosine kinases) }\end{array}$ & $\begin{array}{l}\text { Decreased lung metastasis of CRC (via activation of the } \\
\text { protein tyrosine phosphatase SHP1) and shrank existing } \\
\text { metastases }^{202}\end{array}$ \\
\hline GW3965 & Liver $\mathrm{X}$ receptors & $\begin{array}{l}\text { Inhibited brain metastasis of melanoma and shrank existing } \\
\text { lesions }^{203}\end{array}$ \\
\hline Low-dose paclitaxel & Tubulin & $\begin{array}{l}\text { Decreased lung metastasis of cholangiocarcinoma } \\
\text { (via reduced nuclear import of the calcium-binding }_{\text {protein S100A4) }}^{204}\end{array}$ \\
\hline Selumetinib & MEK & Decreased lung metastasis of TNBC ${ }^{205}$ \\
\hline G2 & Fascin (actin-bundling protein) & Decreased lung metastases of breast cancer ${ }^{206}$ \\
\hline Zileuton & Arachidonate 5-lipoxygenase & Reduced spontaneous metastasis of MMTV-PyMT cells ${ }^{168}$ \\
\hline
\end{tabular}


Table 1 (cont.) | Selected preclinical data for potential anti-metastatic therapies

\begin{tabular}{|c|c|c|}
\hline Agent & Target & Preclinical data ${ }^{a}$ \\
\hline \multicolumn{3}{|l|}{ Peptides } \\
\hline $\begin{array}{l}\text { Bone metastasis-targeting } \\
\text { peptide } 78\end{array}$ & Endoplasmic reticulum chaperone $\mathrm{BiP}$ & $\begin{array}{l}\text { Reduced outgrowth of established lung and bone } \\
\text { micrometastases in an advanced-stage breast cancer model }{ }^{207}\end{array}$ \\
\hline $\mathrm{T} 22$ & CXCR4 (SDF1 chemokine signalling) & $\begin{array}{l}\text { Showed synergy with anti-CTLA- } 4 \text { therapy in reducing the size } \\
\text { of established melanoma metastases }{ }^{208}\end{array}$ \\
\hline Ac-PhScN-NH ${ }_{2}$ & a5 $\beta 1$ integrin (fibronectin receptor) & $\begin{array}{l}\text { Inhibited bone metastasis, disease progression and lung } \\
\text { colonization in a breast cancer model and shrank established } \\
\text { lesions }{ }^{209}\end{array}$ \\
\hline \multicolumn{3}{|l|}{ Immunotherapies } \\
\hline MTDHDNA vaccine & MTDH & $\begin{array}{l}\text { Induced T cell responses and prevented lung metastasis in a } \\
\text { breast cancer model }{ }^{210}\end{array}$ \\
\hline LMP1 DNA vaccine & LMP1 viral antigen & $\begin{array}{l}\text { Inhibited TC-1 lung metastasis in vivo via targeting of EBV } \\
\text { LMPs }^{211}\end{array}$ \\
\hline \multicolumn{3}{|l|}{ Others } \\
\hline Retinoic acid & Retinoic acid receptor (inhibits cell adhesion) & $\begin{array}{l}\text { Inhibited melanoma lung metastasis by inhibiting tumour cell } \\
\text { adhesion to the vascular endothelium and subendothelium }{ }^{212}\end{array}$ \\
\hline IGF trap & IGF1R & $\begin{array}{l}\text { Promoted apoptosis of colon and lung cancer cells in nascent } \\
\text { liver metastases }{ }^{213}\end{array}$ \\
\hline $\begin{array}{l}\text { Ad.dcn (decorin-expressing } \\
\text { oncolytic adenovirus) }\end{array}$ & $\begin{array}{l}\text { Various (results in downregulation of MET, } \\
\beta \text {-catenin and VEGFA) }\end{array}$ & $\begin{array}{l}\text { Systemic delivery shrank established bone metastases of } \\
\text { prostate cancer }{ }^{214}\end{array}$ \\
\hline $\begin{array}{l}\text { Cellax-DTX polymer } \\
\text { (docetaxel-acetylated car- } \\
\text { boxymethylcellulose-PEG } \\
\text { conjugate nanoparticles) }\end{array}$ & $\begin{array}{l}\text { Tubulin (results in selective depletion of activated, } \\
\text { cancer-associated fibroblasts) }\end{array}$ & Decreased development of pancreatic metastases ${ }^{215}$ \\
\hline $\mathrm{N}$-acetylcysteine & Reactive oxygen species (antioxidant) & Inhibited liver metastasis of pancreatic cancer ${ }^{216}$ \\
\hline $\begin{array}{l}\text { NM-NP-CFZ } \\
\text { (neutrophil-mimicking- } \\
\text { nanoparticles containing } \\
\text { carfilzomib) }\end{array}$ & Inflammatory neutrophils & $\begin{array}{l}\text { Prevented early lung metastases and shrank established } \\
\text { metastases in mammary carcinoma models }{ }^{217}\end{array}$ \\
\hline \multicolumn{3}{|c|}{$\begin{array}{l}\text { CDCP1, CUB domain-containing protein 1; CRC, colorectal cancer; CTLA-4, cytotoxic T lymphocyte antigen 4; EBV, Epstein-Barr virus; GPI, glycosylphosphatidy- } \\
\text { linositol; LMP, latent membrane protein; MMP, matrix metalloproteinase; MRTF, myocardin-related transcription factor; MTDH, metadherin (also known as LYRIC); } \\
\text { PEG, polyethylene glycol; SRF, serum response factor; TNBC, triple-negative breast cancer. anless otherwise noted, the intervention was shown to prevent or } \\
\text { delay the development of metastasis. }\end{array}$} \\
\hline
\end{tabular}

with strong correlations with metastasis but not passing the functional tests might serve as biomarkers of a response.

\section{Preclinical modelling}

Effective candidate identification relies on preclinical models that accurately recapitulate the disease pathogenesis in patients and, specifically, the particular process being targeted (that is, initial invasion, extravasation, the development of nascent metastases or metastatic outgrowth). Many commonly used preclinical tumour models are better suited for testing agents that have a direct antitumour effect, often on primary tumours, rather than clinically relevant effects on metastasis ${ }^{51,52}$. For numerous cancer types, the primary lesion can be well controlled by standard therapies (surgery, radiotherapy and/or chemotherapy) - the challenge is to control the onset and growth of secondary lesions.

No single preclinical model exists that wholly reflects metastasis in patients with cancer. Several different preclinical models of the type of cancer under study should be used when testing the anti-metastatic activity of a new drug to account for the diversity of the disease in patients ${ }^{52}$. If a molecularly targeted therapy is to be tested, an ideal preclinical model is one in which the target molecule promotes one or more steps in the metastatic process within that preclinical model. For example, inducible expression of the transcription factor Twist-related protein 1 in mice can drive epithelialto-mesenchymal transition and result in increased metastasis of squamous cell carcinoma ${ }^{53}$. Other examples include prevention of metastases through targeting of SRC expression in orthotopic mouse models of human pancreatic adenocarcinoma ${ }^{54}$ or dual pharmacological inhibition of MET and VEGFR2 (REF. ${ }^{55}$ ) in various tumour models and tumour cell lines, or transgenic mice in which mammary tumour development and metastasis are driven by the expression of HER2 $\left(\mathrm{REF}^{56}{ }^{56}\right.$ or E545K-mutant PIK3CA ${ }^{57}$. Examining drug efficacy in models with complex genetics, including in both metastatic driver and passenger pathways, can also be informative by reflecting the genomic complexity of patient tumours.

The fact that host tissues dictate the extent to which a tumour can metastasize is becoming increasingly evident. The tumour microenvironment is complex, varies extensively in different organs and is influenced by tumour-host cell interactions, physical and metabolic changes, and secreted cytokines, chemokines and growth factors $^{58}$. As well as influencing tumour growth directly, 
these changes alter the capacity of the immune system to recognize and attack tumour cells ${ }^{59}$. These factors must be taken into account when selecting preclinical models in order to ensure that interventions are effective for the tumour type and/or organ of metastatic involvement ${ }^{58}$. For example, in a mouse model of prostate cancer, androgen ablation (by castration) was demonstrated to result in bone loss and enhanced the growth of disseminated tumour cells (DTCs) in the bone; however, detrimental exacerbation of bone metastasis could be overcome by administering bisphosphonates together with androgen ablation ${ }^{60}$.

No in vitro test adequately models the entire metastatic process; therefore, in vivo modelling is essential. Generally, metastasis models consist of either mouse tumours in a syngeneic host, thereby allowing for full engagement of the immune system, or human tumours engrafted into immunosuppressed hosts ${ }^{52,61}$. Mammals other than mice, such as rats ${ }^{62}$, are used occasionally, as are non-mammalian hosts such as zebrafish ${ }^{63}$, Drosophila ${ }^{64}$ and the chick embryo chorioallantoic membrane ${ }^{65}$.

Genetically engineered mouse models (GEMMs) use mice bearing oncogenes that initiate a primary tumour (quite often, multiple primary tumours), which in some cases progress to metastatic disease (for example, the LSL-Kras ${ }^{\mathrm{G} 12 \mathrm{D} /+}$; LSL-Tp53 $3^{\mathrm{R} 172 \mathrm{H} /+} ;$ Pdx1-Cre KPC GEMM used for preclinical studies of both pancreatic cancer prevention and therapy $\left.{ }^{66}\right)$. When murine transplantable tumour models are used, cells can be introduced, preferably at orthotopic sites, to initiate primary tumour growth and subsequent spontaneous metastasis. A major advantage of such mouse metastasis models is the presence of matched stromal tissues that can recapitulate growth factor signalling and anticancer immune responses. Given the obvious and critical role of the host immune system in regulating metastasis ${ }^{58,59,67}$, models of murine tumours in immunocompetent syngeneic hosts should be included in the preclinical testing of any new therapeutic whenever possible.

Metastasis assays can also involve established cancer cell lines or tissues recovered directly from patients undergoing tumour biopsy sampling or resection. As opposed to a cell line that has drifted genomically during long-term culturing, patient-derived xenografts (PDXs) have the important advantage of more closely reflecting the genomic profile of the original tumour. PDXs are also reported to metastasize to the same organs as metastases in the donor patient ${ }^{68-70}$. However, xenograft models obviously lack competent immune regulation (owing to the need to avoid immune-mediated destruction of the transplanted allogeneic tumour cells). The advent of even more severely immunocompromised mice, such as non-obese diabetic-severe combined immunodeficient (NOD-SCID) and NOD-SCIDIL-2-receptor $\gamma$-chain-mutant (NSG) mice, has enabled a higher proportion of tumours to be established in such models, with more circulating tumour cells (CTCs) and metastases. However, in patients, tumours develop despite the presence of a competent immune system by evolving mechanisms to escape immune detection and/or destruction; therefore, various methods of incorporating a human immune system into these severely immunocompromised mice are the subject of active research $^{61}$. CTCs isolated from $10 \mathrm{ml}$ of blood have also been used to generate patient-derived CTC xenograft models (CDXs) and have several advantages over PDX models generated using tumour biopsy samples. These advantages include the development of tumours with a molecular profile generally similar to that of the primary tumour and single CDXs that respond to chemotherapy in the same way as the donor patient's tumour; the ability to generate models for patients with tumours that are not amenable to biopsy sampling or surgery; and the use of a population of tumour cells that has already gained an invasive behaviour, reflecting intra-patient heterogeneity, and that can be used as a surrogate to study metastasis (reviewed in REF. ${ }^{71}$ ). Nevertheless, CDXs do have drawbacks, such as lack of a functional immune system, and can be challenging to establish for some tumour types.

Cell lines and dissociated murine tumours can also be injected haematogenously, intraperitoneally, intrasplenically or by other routes to circumvent primary tumour formation. This scenario might be justified for types of cancer in which initial seeding of tumour cells at distant sites commonly occurs before diagnosis. Such 'experimental metastasis' models offer the advantages of rapid metastatic progression and greater numbers of tumours, which can accelerate study throughput. However, these models might fail to recapitulate the aforementioned capacity of the primary tumour to fashion the premetastatic niche by releasing growth factors, cytokines, proteases and extracellular vesicles ${ }^{14,72,73}$ (FIG. 2).

Models with different sites of spontaneous metastatic dissemination that reflect the patterns of metastasis in patients are a further necessity for preclinical testing. The 4T1.2 mammary cancer model is an example of a mouse model that recapitulates the pattern of metastatic spread of its human counterpart, with the primary tumour generating spontaneous metastases in the lymph nodes, lungs and bones - major sites of metastasis in patients with breast cancer ${ }^{45}$. Clearly, therapies will need to be tailored for metastatic lesions in different organs because the microenvironments of bone, liver and brain, for example, are very different and therefore confer different properties to the tumour cells that successfully colonize these organs. For example, treatment with the RANKL antagonist osteoprotegerin effectively controls bone metastases, but not metastasis to the lungs in a mouse model of metastatic breast cancer ${ }^{74}$.

In general, too few preclinical metastasis models are available to adequately replicate the substantial heterogeneity of metastases in patients. Ideal models should be orthotopic, immunocompetent and able to produce metastases within a few months. Several common types of cancer, including melanoma, breast cancer and prostate cancer, can have a long latency - up to two decades between the initial treatment of the primary tumour and the development of distant metastases. For these cancers, evidence exists for very early dissemination of the tumour cells, even before diagnosis, followed by long periods of dormancy after extravasation into other tissues $^{22,75-80}$ (FIG. 2). These findings and those of other similar studies emphasize the importance of developing 
therapies that can prevent the outgrowth of DTCs or that result in the death of very slowly cycling cells. Preclinical models that mimic this metastatic dormancy phenotype need to be developed to facilitate testing of such therapies and should incorporate current adjuvant treatments, such as hormone therapy for breast or prostate cancer. Current models involve the use of transplantable tumour cell lines with much delayed development of metastatic disease that becomes evident only after an extended time following successful resection of the primary tumour. Examples include the mammary tumour lines D2.OR ${ }^{81}$ and D2.A1-GFP ${ }^{82}$ and HEp3 cells isolated from the lymph node of a patient with head and neck squamous cell carcinoma ${ }^{83}$. Thus, various important features need to be considered when developing preclinical models of metastasis suitable to evaluation of experimental anti-metastatic agents (BOX 1).

\section{Preclinical outcome measures}

Development of metastases in preclinical models can be monitored by non-invasive imaging methods (for example, bioluminescence or MRI), enabling kinetic studies of their development. Standard metastasis models use imaging, histological counts and/or a quantitative measure of metastatic burden ${ }^{45}$ as primary end points, with survival as a secondary end point. Less often, other potentially important end points, such as rates of cell proliferation and apoptosis, microenvironmental alterations or immune infiltration are reported. This approach enables investigation of novel therapies

\section{Box 1 | Validating a potential anti-metastatic agent using preclinical in vivo models}

Animal models are required to provide mechanistic insights into the effects of experimental agents on the entire metastatic process and are conducted primarily in mice. Multiple factors are important to consider when establishing the experimental design if a translational goal is anticipated.

Prevention of metastasis versus shrinkage of existing lesion

Most preclinical metastasis experiments are focused on preventing the initial formation of a metastasis - tumour cells are injected into mice, and the experimental agent is delivered soon after and continuously, with the number and sizes of metastases quantified at the end point. The findings of such studies are more relevant to adjuvant trial designs given the close alignment of aims between the clinical setting and these models - that is, avoiding relapse owing to undetectable disease. Even so, metastatic tumours can be allowed to grow to reflect a more advanced-stage cancer setting.

\section{Spontaneous versus experimental metastasis}

Metastasis models in which neoplastic cells form a primary tumour and subsequently metastasize are the gold standard, but the low number of lesions produced over long periods of time is an important limitation; few such models exist. Other experimental models use haematogenous or other routes of tumour cell delivery and enable interrogation of the final stage of metastasis: metastatic colonization.

The source of tumour cells

Cell lines are easier to use, but long-term culture can result in cell lines that do not accurately recapitulate the actual clinical disease biology. Genetically engineered models generally have low frequencies of metastases and might not reproduce the genomic diversity of human tumours. By contrast, patient-derived xenografts and spheroids have been reported to closely replicate the tumour heterogeneity and evolution and the clinical course ${ }^{70,149}$. Ultimately, the use of multiple models is always preferable.

The animal (typically mice)

Given the increasing importance of immunotherapy, and the immune contributions to responses to standard therapies, use of syngeneic, immune-proficient mice is advisable. Indeed, multiple aspects of the microenvironment are contributors to the metastatic cascade and ideally should be considered in preclinical models.

Site of injection

Subcutaneous models should be avoided. For models of spontaneous metastasis, orthotopic injection of tumour cells is mandatory.

Site of metastasis

The sites of metastasis in the mouse model should reflect the characteristic sites of dissemination associated with the human disease being studied.

Prior chemotherapy or radiation therapy, or concurrent therapy

Any new therapeutic must be given in the context of approved standard treatments. The model can be used to assess whether the new therapy should replace the standard treatment, be combined with it or be used sequentially.

Likely combination therapies

The functional redundancy of the metastatic process ultimately mandates that drug combinations be developed.

Oral or intravenous dosing

When long-term drug administration is required, as in most metastasis prevention studies, oral dosing is imperative unless agents with long half-lives, such as monoclonal antibodies, are under development. Other routes of administration should be considered only if they are used clinically for the cancer type under study.

Pharmacokinetics

Collaborate with a pharmacologist, collect serum samples and generate pharmacokinetic data.

Other end points

Whenever possible, use end points in animal studies that closely reflect clinically relevant end points, such as disease-free survival, quality of life and overall survival, rather than tumour growth curves alone. 
but also the development of drug resistance that is associated with metastasis. Chemoresistance is often considered to be caused by gene mutations that negate the cytotoxic effects of therapy. Surprisingly, diverse metastasis pathways have been functionally implicated in chemoresistance in mouse models (Supplementary Box S1). Mechanistically, reductions in chemosensitivity have often been associated with activation of metastatic pathways that provide survival cues to the tumour cells, enhance proliferative signalling or counter DNA damage ${ }^{84-86}$. These findings uncover an unexpected intersection between metastasis and drug resistance that could lead to rational combinations of anti-metastatic agents and chemotherapies or other cytotoxic agents in clinical trials. These studies offer a provocative translational hypothesis, although careful studies using clinically achievable drug doses, schedules and combinations are needed in multiple preclinical model systems, including both chemotherapy-naive and chemoresistant models.

Another consideration when developing antimetastatic therapies is whether to target tumour cells directly or indirectly by modifying the tumour microenvironment to be more suppressive to tumour growth. A common argument for targeting host cells is their greater genomic stability, which might make the development of drug resistance mechanisms less likely. However, the tumour microenvironment is complex and differs between each organ in which metastases have been established. Although not always adequate to predict metastatic events, preclinical models can help to understand the different host microenvironments and can be used to test therapies focused on site-specific metastasis. The unique microenvironment of bone provides the best example, relating to the aforementioned application of bisphosphonates and denosumab. In preclinical models of breast cancer bone metastasis, responses to bisphosphonates were found to be dependent on menopausal status: ovariectomized mice (mimicking a postmenopausal state) had a greater tumour burden in bone than control mice (mimicking a premenopausal state) but responded well to the therapy, whereas tumour burden in control mice was unaffected ${ }^{87}$. Likewise, in patients with breast cancer participating in the AZURE trial (zoledronic acid in combination with standard adjuvant therapy), a significant improvement in DFS (HR 0.75, 95\% CI $0.59-0.96 ; P=0.02)$ and $\mathrm{OS}(\mathrm{HR}=0.74,95 \% \mathrm{CI} 0.55$ $0.98 ; P=0.04)$ was obtained only in women who had been postmenopausal for $>5$ years at the commencement of the trial ${ }^{88}$.

Development of pharmacodynamic markers indicative of drug activity must also be incorporated into preclinical testing. In clinical trials, traditional end points of radiological tumour responses and survival are increasingly inadequate. Thus, knowledge of whether the drug hits its intended target and how the tumour cells and/or tumour microenvironment respond is of growing importance. When designing experiments in animals, adequate consideration should be given to analyses that are achievable in patients - that is, liquid and/or tumour biopsy-based assessments and imaging. Circulating cellfree tumour DNA (ctDNA), tumour-derived exosomes or CTCs are increasingly common sources of pharmacodynamic markers that can be tested in mice and patients $^{89-94}$.

\section{Comparisons with human tissues}

Evidence of the activity of a prospective target using preclinical models is necessary but is not sufficient: assessment of relevance using clinical samples is imperative. Clinical evidence can be obtained retrospectively by analysing publicly available databases for the transcript of interest and assessing its prognostic and/or predictive value in a specified cohort of patients with cancer that has appropriate follow-up data. Such databases could also be used to identify potential predictive biomarkers for later study in clinical trials. Supplementing analyses with additional information, such as data on inactivation or mutations in genes specifically associated with metastasis, could also help build evidence for a particular target. If adopted, this approach must be used with caution, given that genetic alterations recorded in large databases might provide less robust targets for anti-metastatic agents than for other anticancer therapies because many metastasis pathways involve alterations in gene expression rather than mutations ${ }^{95}$. Any correlations must be confirmed at the protein level in tumour samples from a large number of patients, typically using tissue microarrays. For example, in preclinical models of spontaneous mammary tumour metastasis to bone, restoration of interferon regulatory factor 7 (IRF7) expression in the tumour cells did not alter primary tumour growth but did inhibit bone metastasis ${ }^{44}$. In a retrospective analysis of data from 855 samples from primary breast cancers, high expression of an IRF7 pathway gene signature was associated with reduced bone metastasis of breast cancer (HR 0.63, 95\% CI 0.42-0.93; $P=0.021$ ) but had no prognostic value in predicting metastasis to visceral organs ${ }^{44}$. In tissue array analyses, IRF7 protein was detected in $56 \%$ of primary tumours compared with $17 \%$ of distant metastases and in only $11 \%$ of bone metastases ${ }^{44}$. Prospective analyses can provide very strong evidence for potential therapeutic targets or biomarkers, but in the absence of the relevant cohorts for prospective analysis, appropriately designed prospective retrospective analyses can also generate good levels of evidence ${ }^{96}$.

Associations with non-coding RNA species can be assessed similarly through in situ hybridization in tissue samples. Metastatic biopsy samples are rarer than primary tumour specimens but are important for analyses of biomarker expression in the development of anti-metastatic therapies. Data must be interpreted with consideration of the patient's prior treatments (or lack thereof) in terms of consistency with the proposed clinical setting in which the therapy will be developed.

\section{Drug properties}

The desired pharmacological profile used to guide drug screening necessitates a shift from that typically accepted in an acute tumour shrinkage model to one of chronic, ideally oral administration - with the exception of a monoclonal antibody, for which monthly infusions might be considered feasible - with an appropriate 
risk-benefit profile. Optimization of structure-activity relationships can be used to select lead drug candidates with a pharmacokinetic profile appropriate for chronic use. For example, drugs that have good oral absorption characteristics and effective distribution to target tissues and are poor substrates for metabolic (cytochrome P450) enzymes in the liver (to avoid potential drugdrug interactions) are suited to simple once daily oral administration over months or years.

In the cancer setting, specific characteristics of the target disease, such as the frequency of metastasis to the brain, must also be considered ${ }^{97-99}$. For the example of brain metastasis, drug properties that increase bloodbrain barrier permeability and reduce the potential for membrane efflux transporter elimination might be a priority. Toxicological structure alerts (toxicophores) need to be avoided in the development of any drug that is to be dosed chronically, particularly in elderly or frail patients who are likely to present with comorbidities, including reduced renal and liver function, or in patients who have previously been treated with multiple lines of anticancer therapy and are therefore likely to have a poor performance status. Avoidance of drugs that have high risk of hERG voltage-gated potassium channel blockade, which is associated with potentially fatal cardiotoxicity, is a special case in point ${ }^{100}$. Other pharmacological features associated with on-target and off-target toxicities also need to be avoided, particularly the generation of reactive metabolites that can cause carcinogenicity and nonspecific cytotoxicity.

\section{Clinical drug development Overview}

Designing clinical trials and identifying end points that reflect the prevention of metastatic disease and can meet regulatory authority expectations for evidence of clinically significant benefit are arguably the most critical barrier to the development of new anti-metastatic agents. Measuring a clinically meaningful outcome in a realistic time frame, without requiring the recruitment of overwhelming patient numbers, is a key aim. The paucity of putative anti-metastatic agents being tested in clinical trials bears testament to these barriers.

Chemotherapies, hormone therapies, molecularly targeted agents, immunotherapies and various combinations thereof have produced responses and prolonged progression-free survival (PFS) and OS in the metastatic setting but are insufficient to achieve cure in most patients. Some of these same treatments have prevented or delayed the development of overt metastatic disease in some (but not all) patients when administered to those without detectable metastases but in whom micrometastases are suspected. Examples include the use of combination chemotherapy in the perioperative setting for bladder cancer ${ }^{101,102}$ and adjuvant tamoxifen for breast cancer ${ }^{103}$. This scenario has led to a drug development pathway in which therapies likely to be effective in eliminating micrometastases are progressed to the adjuvant setting only after they first demonstrate antitumour effects in patients with advanced-stage disease. Such an approach also assumes that the biology of the metastases is similar to that of the primary tumour, which is not universally true. This drug development paradigm is further reinforced by the need to manage the very large risks associated with trials in the adjuvant setting (for example, relating to the need for large cohorts, healthier populations and long durations for data maturity; the high costs; and the lack of safety data in patients with anticipated long-term survival) by first demonstrating activity and acceptable toxicity in trials involving smaller numbers of patients with more rapidly attainable end points. In other words, demonstrated efficacy in patients with pre-existing metastases has traditionally been the obligate gateway to adjuvant trials of interventions for metastasis prevention.

The ability to delay or prevent metastases has the potential to enormously improve the survival durations of patients with cancer and could even lead to cures. However, the opportunity presented by anti-metastatic drugs cannot be explored adequately using conventional drug development pathways because drugs without cytotoxic or clinically meaningful cytostatic effects in the patients with overt metastatic disease will never advance to adjuvant trials. This point is central to the rethinking of clinical trial designs for metastasis prevention, with the clinical use of potential anti-metastatic drugs falling into three possible scenarios.

Occult micrometastatic disease. A practical definition of micrometastatic disease is that which is suspected to be present at the time of treatment of the primary tumour but is not evident using conventional imaging and clinical examination. Micrometastases can sometimes be detected by other methods, such as in bone marrow aspirates using flow cytometry ${ }^{104}$, biochemical techniques (such as rising serum PSA levels in patients with prostate cancer ${ }^{105}$ ) or molecular assays for tumourderived DNA (typically ctDNA in blood samples ${ }^{106}$ ). However, the predictive power of some of these tests is debatable, with questions remaining regarding their sensitivity and specificity ${ }^{107}$. Prospective randomized clinical studies in specific disease settings are required to examine the utility of these detection methods and thereby validate their use in guiding treatment decisions. Patients with micrometastasis can be identified across many, if not most, types of cancer, providing important new opportunities for anti-metastatic therapy. Occult micrometastatic disease might either be actively growing or dormant and how this difference might affect the choice and timing of therapy remains unclear. The detection of rising PSA levels in patients with no other evidence of disease after prior treatment of prostate cancer can be used as a platform for metastasis prevention (NCT03119857), although many trials simply use PSA metrics as a primary end point of treatment efficacy.

Tumours that cannot be removed surgically. In general, the aim of cancer surgery is to remove the primary tumour in order to obtain optimal local disease control and prevent the subsequent development of metastatic disease. Often, however, tumour resection is not feasible - despite the absence of metastatic disease - because of the anatomical location of the tumour or the likely 
inability of the individual patient to tolerate the proposed surgery (for example, owing to an insufficient respiratory reserve to endure pneumonectomy or insufficient fitness for prolonged anaesthesia). In practice, management of these patients presents a substantial clinical challenge. The use of drugs to prevent metastatic spread in addition to therapy to achieve local disease control (for example, by chemoradiation of locally advanced pancreatic cancer) is a potential approach to overcoming this challenge. This strategy could also be an alternative to tumour resection for patients in whom the morbidity and mortality associated with surgery would be better avoided. Another option for such patients arises from the emerging realization of the abscopal effects of radiotherapy - that local radiotherapy can lead to immune-mediated destruction of tumours outside the irradiated field ${ }^{108}$. Several clinical trials (NCT03323424, NCT03396471 and NCT02992912) are now testing the combination of local radiotherapy with immunotherapy to exacerbate the damage to overt metastatic lesions outside the radiation field ${ }^{109,110}$. If this hypothesis is proven, then the abscopal effect could potentially be harnessed for the management of micrometastatic disease to effect cures in future trials.

Patients at high risk of invasive malignancy. Metastasis is rare in the absence of an invasive primary tumour; thus, a strong clinical rationale exists for eliminating cancer in its pre-invasive state to prevent disseminated disease. Patients with discrete pre-invasive lesions might undergo major local therapy; for example, patients with non-invasive tumours of the bladder often undergo cystectomy ${ }^{111}$, and those with breast ductal carcinoma in situ can undergo mastectomy ${ }^{112}$. Even for some pre-cancerous conditions, the established organ-wide risk of pre-invasive disease (and thus malignant transformation) can warrant preventive surgery (such as pan-colectomy for familial polyposis) or regular surveillance with a view to major surgery (such as Barrett oesophagus). In some cases, complete removal of all at-risk tissue is not possible (for example, in patients with urothelial carcinoma in situ). Indeed, existing treatments are already used to reduce the risk of progression in patients with pre-invasive neoplasia (such as intra-vesical Bacillus Calmette-Guerin (BCG) immunotherapy for those with urothelial carcinoma in situ) or a known risk of developing cancer (for example, tamoxifen for women with an inherited risk of breast cancer owing to $B R C A$ mutations). However, these approaches often have a disappointing effect on survival outcomes ${ }^{113,114}$, and the agents themselves might not target the pathways that most potently drive the invasive phenotype. Furthermore, continuous long-term administration of potentially toxic agents can be required to maintain the chemopreventive effect, which might not be feasible; for example, chemoprevention with retinoids in patients with pre-malignant head and neck tumours has been limited by poor tolerability (with the majority experiencing cheilitis, dry skin and conjunctivitis ${ }^{115}$. Consequently, the opportunity to develop rationally targeted, more efficacious and better tolerated systemic therapies in such patient populations is attractive.
In this scenario, anti-invasion therapies might be the most useful anti-metastatic approach.

\section{Challenges in clinical trial design}

A dominant problem in each of the preceding scenarios is that intervention is likely to be required years in advance of the clinically important event (predominantly metastatic relapse or death). This latency presents substantial challenges for the design of statistically powered clinical trials that meet the regulatory standards for evidence. The main implications are both economic and clinical. Economically, the costs of trials with large cohorts and long follow-up durations are prohibitive, and the predicted return on investment is restricted by the potentially limited time remaining on a patent. Indeed, many current adjuvant therapies have come into routine use only after or near the time of patent expiry. For this reason, such studies are rarely industry funded. Clinically, the imperative is to make more rapid progress than this scenario allows.

The end points traditionally used in oncology clinical trials present an additional challenge. End points for determining antitumour efficacy in patients with advanced-stage disease continue to be based on conventional outcome measures including the objective response rate (ORR) based on reductions in tumour dimensions on cross-sectional imaging of established metastases, PFS and/or OS. Adjuvant trials typically have a primary end point of DFS. These indicators of efficacy either present a specific challenge to the timely development of anti-metastatic therapy or are not applicable in the absence of lesions that are detectable on imaging. Biomarkers that enable the detection of disease progression earlier than is possible through imaging of new metastatic lesions are clearly desirable; however, the use of such markers might require prospective validation, compounding the time and financial requirements.

With these challenges in mind, several ways forward are discernible. Owing to the plethora of potential drug candidates and the high costs of late-phase clinical studies, early clinical development must be maximized as follows. Anti-metastatic agents are unlikely to be substantially different from any other candidate anticancer drug in this regard, but early go versus no-go decisions will need to be based on robust mechanistic and other pharmacodynamic biomarkers rather than on the typical evidence of objective tumour responses in patients with advanced-stage disease. Given the essential favourable safety profile of these anti-metastatic drugs, efficient early phase clinical studies could feasibly be conducted in healthy volunteers; thus, the identification of biomarkers that are evaluable in non-malignant tissues will be of crucial importance.

The appropriateness of the novel end points will need to be supported by community-derived data providing compelling evidence that regulatory authority requirements will be met. By their nature, the novel end points are likely to be context-specific. Nevertheless, examples are available of accepted surrogate end points that have been used successfully in clinical development of drugs targeting pre-invasive pathways. These examples include the use of progression from early stage to stage 
T2 disease in establishing the role of intravesical BCG for the treatment of high-risk non-muscle-invasive bladder cancer ${ }^{113}$ and the use of a reduction in the numbers of polyps in the development of celecoxib for chemoprevention in patients with familial polyposis coli ${ }^{116}$. Early approval, on the basis on surrogate end points, will then need to be followed by confirmation of the clinical benefit in registry-type post-marketing studies.

In February 2018, the FDA also accepted the surrogate end point of metastasis-free survival in the first registration of apalutamide for the treatment of non-metastatic CRPC ${ }^{117}$. However, this end point was controversial ${ }^{118}$ because the use of conventional imaging (with isotope bone scans and CT) to establish the metastasis-free state will certainly result in failures to identify metastases that might be detectable using novel imaging modalities, such as whole-body MRI ${ }^{119}$ or ${ }^{68} \mathrm{Ga}$-prostate-specific membrane antigen-PET ${ }^{120}$. The use of these novel imaging techniques is unlikely to have changed the outcome of the trial (an imbalance between the arms in the incidence of MRI-detectable or PET-detectable metastatic disease seems unlikely); however, if patients had disease staging using these alternative, more sensitive imaging modalities, the control intervention (placebo alone) would have been inappropriate for the subset of patients with upstaging to metastatic disease, and thus the true therapeutic value of apalutamide might have been overstated. Hence, completion of dedicated studies to identify the true incidence of metastatic disease in particular patient populations might be necessary before such end points can be used to explore drugs with the specific aim of preventing metastasis.

Secondary prevention studies of metastasis are ongoing. In one such study (NCT03190967), patients with brain-metastatic breast cancer are being randomly assigned to receive metastasis prevention with the antibody-drug conjugate trastuzumab emtansine alone or in combination with metronomic temozolomide, with the primary objective of extending survival without new brain metastasis ${ }^{121}$. Similar end points are being explored in patients randomly assigned to a metastasis preventive strategy or control treatment after surgery for liver-limited metastatic colorectal cancer (for example, NCT03326791 and NCT00394992). Novel liquid biopsy assays of circulating tumour components, including ctDNA and CTCs, could also offer important opportunities for the development of biomarkers as new surrogate end points or intermediate markers to reduce the risk and accelerate the progress of anti-metastatic drug development (see the 'Regulatory and registration pathways' section $)^{91,122-126}$.

Selecting populations at particularly high risk of having an early clinical event at a somewhat predictable rate for inclusion in first proof-of-concept trials - similar to the enrolment of patients at high risk of metastatic recurrence in the aforementioned studies of secondary metastasis prevention strategies - is a key approach to accelerating the development of anti-metastatic drugs. A wealth of literature is available describing prognostic factors that can help to identify such patients ${ }^{127,128}$; however, few studies have been completed prospectively, have incorporated independent validation cohorts or have used methodologies that can be accurately repeated and certified in diagnostic laboratories. The magnitude of metastatic risk is also an important consideration. Whether clinicians will feel comfortable designing a trial in a population with a $30 \%$ or $50 \%$ risk of metastasis over a defined period is debatable. The discovery of novel biomarkers that can be used to estimate the risk of micrometastases is a research area that has not been adequately addressed to date, although the use of ctDNA-based approaches to molecularly define minimal residual disease (MRD) might provide opportunities in this regard. Subgroups of patients with an especially high risk of early distant metastases are clearly identifiable. Examples include those with limited-stage small-cell lung carcinoma ${ }^{129}$, locally advanced pancreatic ductal adenocarcinoma ${ }^{130}$ or colorectal cancer and resectable liver metastases ${ }^{131}$. These disease settings are attractive both commercially and clinically, although reliance on such populations for early go versus no-go decisions holds the risk that effective anti-metastatic drugs will be prematurely discarded. Specifically, these high-risk populations might not display any degree of micrometastatic dormancy. Furthermore, these patients do not present an opportunity to explore drugs targeting pre-invasive targets.

Another way to improve the efficiency of late-stage drug development is to include only patients with disease in which the aberrant pathway being targeted is known to be active. This approach has other clear advantages to patients in that it avoids exposure to the toxicities of such drugs in those who are unlikely to benefit and enables their prioritization to receive other treatments. Indeed, this concept of precision medicine is rapidly becoming mainstream in all areas of anticancer drug development. To enable application of these principles to the development of anti-metastatic drugs, a full understanding of the link between the therapeutic target and the relevant subpopulation is vital, given that metastases are likely to have more complex and different drivers than those of the primary tumour. Heterogeneity between individual metastases adds an additional layer of complexity. Moreover, clinically applicable diagnostics should be developed in parallel with the drugs themselves. For some patients, however, time is of the essence in deciding on the next therapy; thus, a requirement to schedule biopsy sampling and evaluate the specimen in an accredited laboratory might present difficulties, particularly in the secondary metastatic setting, in which the oncologist and patient want to decide quickly on the next line of therapy (or trial participation). Conversely, in the postoperative adjuvant setting, this time delay might not be clinically relevant while the patient recovers from the primary surgical management. Similarly, when the aim is to evaluate the novel agent in a maintenance setting, the patient first needs to complete conventional postoperative systemic anticancer therapy.

Drugs that specifically target the processes of metastasis have the potential to transform the care of the majority of patients with solid tumours, although such agents are unlikely to be used as their sole therapy. One can envisage that treatment of the primary tumour and 
standard-of-care adjuvant therapy will remain the initial interventions, followed by potentially lifelong maintenance therapy to prevent the growth of pre-existing (micro)metastases and/or to prevent further spread of metastases. Thus, one challenging but necessary aim of clinical trials will be to establish the optimal combination and sequencing of these therapies with other modalities including surgery, radiotherapy, chemotherapy, molecularly targeted therapy and/or immunotherapy. The need to address the long-term toxicities of these drugs given the likely chronic duration of therapy is also essential.

\section{Regulatory and registration pathways}

Many promising mechanistic biological targets exist for metastasis prevention strategies (TABLE 1); however, clear and feasible regulatory pathways for anti-metastatic agents are lacking. Specifically, the lack of surrogate end points appropriate for determining clinical efficacy at an early stage of drug development makes anti-metastatic agents unattractive to developers when compared with drug classes with rapid and proven pathways to registration.

A primary disincentive for anti-metastatic agents is the de facto use of time-to-event end points, such as PFS or OS, to assess efficacy in early stage clinical research. These end points present a far greater logistical and financial hurdle than the short-term ORR end points used to assess almost all newly registered oncology agents. In the period of 2006-2016, the FDA approved 180 oncology drugs (new and supplemental registrations), including 41 accelerated approvals: most accelerated approvals $(n=37)$ were based on ORRs, with the remaining 4 approvals based on PFS using assessment criteria defined by indication-specific working groups ${ }^{132}$. The RECIST alone were used in the majority of studies that led to accelerated approvals. The RECIST and working group-derived criteria are standardized, welldefined and provide a rapid readout of objective tumour responses or disease progression ${ }^{5}$, whereas assessment criteria appropriate for anti-metastatic treatments are not standardized or clearly defined. These limitations are particularly problematic because the existing criteria designed for assessing objective responses or progression of solid tumours are unlikely to be of any use in assessing anti-metastatic agents that might have little effect on primary tumours; additionally, in most cases, the patient will not have any detectable disease at the time of treatment with such drugs.

In the absence of regulatory precedent or standardization, individual product developers will be required to explore new surrogate end points without certainty that regulators will accept their clinical relevance. Fortune rarely favours the brave in drug development. Even with a significant increase in biologically relevant bonemetastasis-free survival durations (4.2 month prolongation versus placebo, HR $0.85 ; P=0.028$ ) in 1,432 men with CRPC at high risk of developing bone metastases, the FDA denied an application for expansion of denosumab use to an anti-metastatic indication in this disease $\mathrm{e}^{133}$. The regulator cited that it was "unclear whether an improvement in bone-metastasis-free survival alone in patients with CRPC at high risk of bone metastases is an adequate measure of clinical benefit in support of new labelling claims for a new patient population" (ODAC Briefing Document BLA 125320/28 Denosumab (XGEVA), 2012, FDA $)^{133}$. This example underscores the risk involved in developing anti-metastatic agents and highlights the need to complete robust validation of putative new end points concurrently with the clinical development of such agents ${ }^{134,135}$.

Many other surrogate end points and biomarkers specific to metastatic progression have been considered, including the use of time-to-metastasis end points in patients at high risk of metastasis ${ }^{121}$, residual CTCs, ctDNA, DTCs and circulating tumour exosomes ${ }^{91}$. However, turning these end points and biomarkers into validated surrogates that regulators will endorse as the basis for accelerated approvals will require a collaborative approach to overcome the limitations of novel end points proposed by individual sponsors on an ad hoc basis ${ }^{125}$. These limitations include the high cost of biomarker development, the work required for refinement and standardization of test methods and the requirement for validation across comparable data sets and in sufficiently large numbers of the target population to support statistically robust conclusions ${ }^{136}$.

Plasma ctDNA profiling has demonstrated promise in detecting MRD and discriminating patients with and without eventual clinical recurrence following surgery and/or adjuvant therapy, notably in breast cancer $^{123,124,126,137}$ and non-small-cell lung cancer ${ }^{122}$. These findings emphasize the potential use of ctDNA profiling to select patients at high risk of relapse following completion of adjuvant therapy for add-on anti-metastatic strategies, although these approaches remain in the research setting. Monitoring treatment response to immunotherapy using ctDNA-based liquid biopsy approaches might also be feasible ${ }^{93}$. Prospective, randomized trials are required to test whether persistent or rising ctDNA can be used as a surrogate for adjuvant therapy, alongside development of standardized workflows enabling clinical implementation. The implementation of such a strategy is illustrated by the accelerated FDA approval of blinatumomab in 2018 for the treatment of patients with B cell precursor acute lymphoblastic leukaemia who are in first or second complete remission but have an MRD burden $\geq 0.1 \%$, which occurred on the basis of an end point relating to the achievement of undetectable MRD using a high-sensitivity detection assay after one cycle of treatment ${ }^{138,139}$. In addition, a Clinical Laboratory Improvement Amendments (CLIA)-registered duplex ctDNA blood test for colorectal cancer recurrence has been available in the USA since 2016 for surveillance of patients after tumour resection ${ }^{140}$.

The lack of fixed criteria for drug developers is an unacceptable disincentive, the effects being sadly evident in the lack of any new chemical entity initially registered for the prevention of metastasis. We urge regulatory agencies to work with researchers, drug developers and statisticians to identify and define guidelines for surrogate end points in order to encourage development of this under-represented category of oncology drugs. 
Given that some anti-metastasis agents might be used in a preventive setting, the safety profile of such an Investigational Medicinal Product (IMP) will have to be much cleaner than those of standard anticancer agents. Proceeding to clinical trials even in the absence of adequate preclinical efficacy models might be acceptable, although a strong rationale will be needed to support long-term administration of the IMP in a healthier population of patients with cancer. The acceptable safety profile will be different for IMPs aimed at suppressing and/or eradicating established metastatic lesions. The benefit-risk assessment should include a detailed discussion of standard treatments, the temporal relationship with standard therapy (neoadjuvant, adjuvant or other applications), proposed safety monitoring and risk-minimization strategies; the potential for drugdrug interactions and risks associated with possible delaying of standard therapy should be addressed.

Development of new biomarkers as surrogate end points should be guided by correlations with previously validated end points and/or clinically relevant parameters (reviewed in REF. ${ }^{141}$ ). Companion diagnostics can be developed in parallel to the IMP and used for exploratory purposes. Once validated, the diagnostic can be used to dictate patient eligibility for trial participation and/or treatment assignment. In the USA, the FDA has provided guidance on validation of biomarkers for use in clinical development ${ }^{142}$, and in the European Union (EU), the European Medicines Agency has released guidance for biomarker analysis of clinical trial samples ${ }^{143}$.

\section{Box 2 | Summary of recommendations for the development of anti-metastatic therapies}

\section{Target identification and preclinical models}

- Clear evidence and understanding of the functional relevance and biological activity of the proposed target in metastasis in humans are essential.

- Preclinical models must reflect the disease pathogenesis in patients; for example, the sites of metastasis should encompass the same or similar organs, and the target (or equivalent target in the model) must have the same role as in the human setting. Multiple models should be used.

- Experimental conditions should be designed to reflect the standard of care in the clinical setting and the desired route of treatment administration.

- Careful consideration should be given to the involvement of the immune system and role of chemoresistance within the models used.

\section{Drug discovery and preclinical development}

- Anti-metastatic drugs are likely to be given via the oral route repeatedly over a prolonged period of time (perhaps years), necessitating a shift from the risk-benefit profile of traditional anticancer agents towards one with a considerably lower level of risk; the absorption, distribution, metabolism and excretion (ADME) profile of the drug should reflect this necessity.

- Drugs that have liver metabolism liabilities and/or common drug-drug interactions, undergo enzymatic hydrolysis or are substrates of membrane efflux transporters should be avoided.

- The pharmacokinetic parameters of the drug must be optimized to enable high levels of target exposure, including a high dissolution rate and good solubility and permeability.

- Lead clinical drug candidates should be tested in several different and, ideally, genomically complex models to account for diversity of the disease in patients.

- Pharmacodynamic markers need to be developed that reflect drug activity and can be translated to the clinical setting.

- Toxicology readouts should generally be cleaner than that often accepted for anticancer agents; for example, the drug should not result in blockade of $h E R G$ voltage-gated potassium channels nor generate reactive metabolites that can lead to downstream carcinogenicity or nonspecific toxicity.

\section{Clinical development}

- Given the potential ethical issues surrounding the testing of experimental agents with an anti-metastatic mechanism, specifically relating to the very limited possibility of clinical benefit in patients with advanced-stage cancer, studies involving healthy volunteers should be considered as a first step in clinical testing.

- Early clinical development (ideally phase I expansion cohorts) should be focused on demonstrating proof of biological concept using validated pharmacodynamic markers relevant to the target and drug being tested; if healthy volunteers are enrolled in clinical trials, these markers must be measurable in non-malignant tissues and/or in blood.

- Any companion diagnostics that might have to be developed in parallel to the experimental therapeutic and Conformité Européene (CE) marked (indicating a product that meets European Union (EU) safety, health or environmental requirements and complies with EU legislation) can be used to guide treatment and/or trial enrolment decisions.

- Depending on the mechanism of action of the agent being tested, window of opportunity studies could also be considered during early phase clinical development to enable direct assessment of the biological mechanism in established primary or metastatic tumours.

- Alternate surrogate measures of clinical benefit, beyond traditional radiological criteria based on tumour shrinkage, are needed and will be dependent on the disease and setting being investigated; potential examples include end points based on time to appearance of new lesions and/or secondary lesions or levels of circulating tumour cells or cell-free tumour DNA.

- Initial proof-of-concept clinical studies can be conducted in selected patient populations at high risk of an early clinical event, such as a new metastasis. Thus, before and during clinical development, expert advice from oncologists with experience working with such patient groups should be sought. Moreover, the aberrant pathway under assessment should be confirmed as being active in this population.

- In light of the points above, clinical development timelines longer than those associated with traditional anticancer drugs should be anticipated; early approval based on surrogate outcomes should be a key goal, when possible.

\section{Regulatory pathways}

- Ensure that a strong rationale is provided in regulatory submissions to support the long-term administration of the anti-metastatic agent in a population of healthier patients with cancer.

- Given the current absence of regulatory precedent or standardization, exploration of new surrogate end points should be discussed with the appropriate regulator before embarking on and during clinical drug development.

- If approval is given on the basis of short-term surrogate end points, a requirement to complete 'sales revenue-funded' phase IV confirmatory studies with larger cohorts, longer follow-up durations and more traditional end points, such as overall survival, should be anticipated.

- We urge regulatory agencies to work together with researchers, drug developers and statisticians to define guidelines on surrogate end points to encourage development of this complex but high-potential category of oncology drugs. 

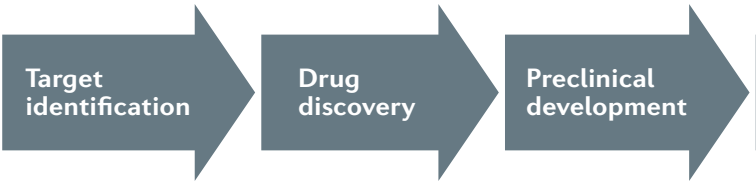

Phase I

clinical trials
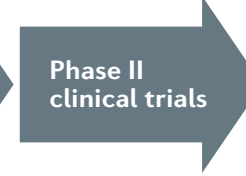

clinical trials

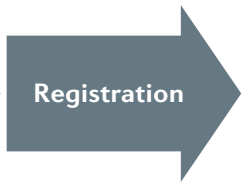

Translation

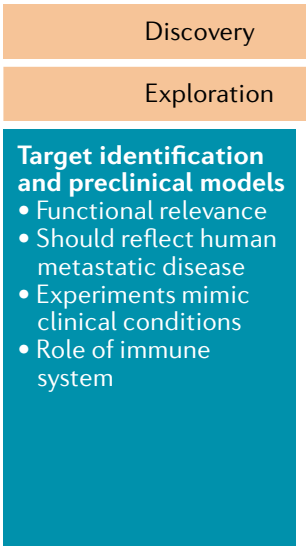

Drug discovery and preclinical development

- Oral drug

- Risk-benefit profile appropriate

for chronic dosing

- Active in several different and

- Active in several different
relevant in vivo models

- Develop relevant PD markers

- Optimized PK/PD profile

(Accelerated) clinical programme
- Phase I studies focused on improved
safety, proof of biology (PD characteristics)
and PK profile in healthy volunteers; PD
characteristics should be measureable in
non-malignant tissue and/or blood
- Consider window-of-opportunity studies in
patients with cancer or at-risk populations
- Efficacy initially measured using surrogate
end point in patients (identified using
CE-marked diagnostics?)
- Be prepared for longer development
timelines, or aim for approval based on
surrogate outcomes
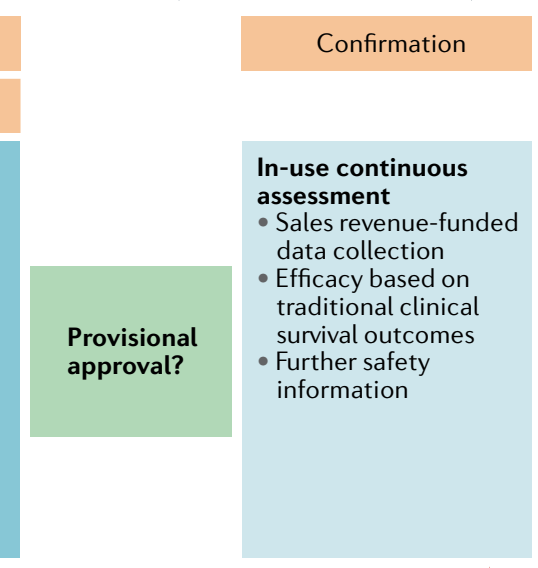

Confirmation

Phase III and/or

phase IV clinical

trial data

Multidisciplinary collaboration to identify and validate new targets, predictive and surrogate biomarkers and end points

Fig. 3 | Development pathway for anti-metastatic agents. The general process for development of anti-metastatic agents has the same fundamental basis as that used in the development of drugs with a direct antitumour mechanism of action, with some special considerations as highlighted in the figure and described as follows. In target identification and preclinical development, special consideration must be given to the functional relevance of the models being used, which should reflect human metastatic disease as much as possible; the role of the immune system in metastasis is a critical factor. The experimental conditions should also mimic those of the clinical setting. Drug discovery and subsequent preclinical testing strategies need to be designed to account for the fact that, in most cases, the anti-metastatic therapy under development will be given chronically in a healthier population of patients, such as those that have been cured of their primary disease but are at high risk of developing secondary tumours, necessitating oral administration and a riskbenefit profile lacking key toxicity liabilities. Other considerations, such as activity in several different preclinical models, an optimized pharmacokinetic (PK) profile and development of pharmacodynamic (PD) markers suitable for use in the clinic, are common to all cancer drug discovery and development programmes. Given the favourable risk-benefit profile necessary for anti-metastatic agents, an accelerated development approach can be taken by conducting initial phase I studies in healthy volunteers rather than the classical populations with advanced-stage cancer. The key aims of these studies are to determine the safety, PK profile and PD characteristics (ensuring the putative biomarkers developed can be measured in non-malignant tissues) in order to provide an early go or no-go decision point and ensure that the drug has the intended biological effects. To gain rapid biological proof of concept in patients with cancer, windowof-opportunity studies, in which a dose of the anti-metastatic agent is given before surgery to examine PD effects, can be considered. If validated surrogate end points of clinical efficacy are available, these can be used to substantially reduce development timelines and, provided agreement has been reached with appropriate regulatory bodies, support provisional approval. If successfully executed, this regulatory strategy will avoid the protracted clinical development timelines that are one of the greatest barriers to the development of anti-metastatic drugs. Provided that provisional approval is given, regulatory bodies will require further in-use continuous assessment, typically in confirmatory phase IV studies that can be funded using ongoing sales revenue. The aim of these larger-cohort and much longer duration clinical trials is to confirm that a pre-defined level of clinical benefit is achieved according to more traditional outcomes, such as overall survival. If provisional approval has not been given by regulators, then costly (in terms of both finance and time) randomized controlled phase III studies in large cohorts will be necessary to gain approval on the basis of standard clinical outcome measures. CE, Conformité Européene.

For marketing any test in the EU, Conformité Européene (CE) marking is required, indicating that the product meets EU safety, health or environmental requirements and compliance with EU legislation.

\section{Conclusions}

Survival outcomes of patients with cancer have steadily improved since the advent of treatments including surgery, chemotherapy, radiotherapy, molecularly targeted therapies and immunotherapy. Nevertheless, in most patients who die of cancer, death is directly attributable to metastasis and not to the primary tumour.
We have highlighted that development of effective therapies to treat and/or prevent metastatic disease requires a marked shift from the standard drug discovery and development paradigm.

Crucially, drug discovery programmes aimed at developing agents that specifically target metastasis should take into consideration the challenges and recommendations proposed herein; to facilitate such studies, we have provided summaries of our recommendations (BOX 2) and overall development pathway (FIG. 3). Careful application of these lessons, learned from past failures, should maximize the probability of success in 
the development of this drug class. Regardless, continued discussion of these issues is warranted.

Indeed, considerable challenges remain, not least regarding the currently limited ability to detect preexisting micrometastatic disease at first diagnosis. Establishment of new detection methods such as the Metas-Chip approach ${ }^{144}$, which uses a microelectronic biochip to detect micrometastasis using small-volume tumour and lymph node samples, will be needed. This method requires biopsy samples consisting of live cells and is based on the principle of detecting the migratory behaviour of tumour cells via their invasive capacity to retract single human umbilical vein endothelial cells from electrical sensing traps. Clear limitations of such assays include the logistical challenges associated with obtaining high-quality live biopsy material in the standard hospital setting and the high probability of no tumour cells being present in the tissue sample. The development of enhanced imaging techniques with greater resolution and sensitivity than those currently available, and their translation to widespread clinical application, will also be vital. Some examples under investigation include high-contrast fluorescence detection ${ }^{145}$, multispectral optoacoustic tomography ${ }^{146}$, shortwave infrared emitting nanoprobes ${ }^{147}$ and novel MRI contrast agents ${ }^{148}$. An ability to detect micrometastases, in addition to primary tumours and macrometastases, using these and other approaches will ensure that patients can be enrolled in clinical trials and assigned to appropriate treatment regimens.

Given the inherent additional complexity, timelines and potential cost of developing anti-metastatic agents, the support of regulators and the pharmaceutical industry will be crucial for future success. If this support can be provided, the potential improvements in the welfare of patients with cancer cannot be understated.

\section{Published online 4 December 2018}

1. Fidler, I. J. \& Kripke, M. L. The challenge of targeting metastasis. Cancer Metastasis Rev. 34, 635-641 (2015).

2. Spano, D., Heck, C., De Antonellis, P., Christofori, G. $\&$ Zollo, M. Molecular networks that regulate cancer metastasis. Semin. Cancer Biol. 22, 234-249 (2012).

3. Vreeland, T. J. et al. Gaining ground on a cure through synergy: combining checkpoint inhibitors with cancer vaccines. Expert Rev. Clin. Immunol. 12, 1347-1357 (2016).

4. Steeg, P. S. Targeting metastasis. Nat. Rev. Cancer 16 201-218 (2016)

5. Therasse, P. et al. New guidelines to evaluate the response to treatment in solid tumors. European Organization for Research and Treatment of Cancer, National Cancer Institute of the United States, National Cancer Institute of Canada. J. Natl Cancer Inst. 92, 205-216 (2000)

6. Eisenhauer, E. A. et al. New response evaluation criteria in solid tumours: revised RECIST guideline (version 1.1). Eur. J. Cancer 45, 228-247 (2009).

7. Pachmayr, E., Treese, C. \& Stein, U. Underlying mechanisms for distant metastasis - molecular biology. Visc. Med. 33, 11-20 (2017).

8. Caswell, D. R. \& Swanton, C. The role of tumour heterogeneity and clonal cooperativity in metastasis, immune evasion and clinical outcome. BMC Med. 15 , 133 (2017).

9. Hosseini, H. et al. Early dissemination seeds metastasis in breast cancer. Nature 540, 552-558 (2016).

10. Werner-Klein, M. et al. Genetic alterations driving metastatic colony formation are acquired outside of the primary tumour in melanoma. Nat. Commun. 9 , 595 (2018).

11. Marshall, J. C. A. et al. Effect of inhibition of the lysophosphatidic acid receptor 1 on metastasis and metastatic dormancy in breast cancer. J. Natl Cancer Inst. 104, 1306-1319 (2012).

12. Avigdor, B. E. et al. Mutational profiles of breast cancer metastases from a rapid autopsy series reveal multiple evolutionary trajectories. JCl Insight 2, 96896 (2017).

13. Puram, S. V. et al. Single-cell transcriptomic analysis of primary and metastatic tumor ecosystems in head and neck cancer. Cell 171, 1611-1624 (2017).

14. Kaplan, R. N. et al. VEGFR1-positive haematopoietic bone marrow progenitors initiate the pre-metastatic niche. Nature 438, 820-827 (2005).

15. Peinado, H. et al. Pre-metastatic niches: organ-specific homes for metastases. Nat. Rev. Cancer 17, 302-317 (2017).

16. Nielsen, S. R et al. Macrophage-secreted granulin supports pancreatic cancer metastasis by inducing liver fibrosis. Nat. Cell Biol. 18, 549-560 (2016).

17. Costa-Silva, B. et al. Pancreatic cancer exosomes initiate pre-metastatic niche formation in the liver. Nat. Cell Biol. 17, 816-826 (2015).

18. Sosa, M. S., Bragado, P. \& Aguirre-Ghiso, J. A Mechanisms of disseminated cancer cell dormancy: an awakening field. Nat. Rev. Cancer 14, 611-622 (2014).
19. Naumov, G. et al. Ineffectiveness of doxorubicin treatment on solitary dormant mammary carcinom cells or late developing metastases. Breast Cancer Res. Treat. 82, 199-206 (2003)

20. Weaver, V. M. et al. $\beta 4$ integrin-dependent formation of polarized three-dimensional architecture confers resistance to apoptosis in normal and malignant mammary epithelium. Cancer Cell 2, 205-216 (2002).

21. Sethi, T. et al. Extracellular matrix proteins protect small cell lung cancer cells against apoptosis: a mechanism for small cell lung cancer growth and drug resistance in vivo. Nat. Med. 5, 662-668 (1999).

22. Ghajar, C. M. et al. The perivascular niche regulates breast tumour dormancy. Nat. Cell Biol. 15, 807-817 (2013).

23. Curran, S. \& Murray, G. I. Matrix metalloproteinases in tumour invasion and metastasis. J. Pathol. 189, 300-308 (1999)

24. DeClerck, Y. A. \& Imren, S. Protease inhibitors: role and potential therapeutic use in human cancer. Eur. J. Cancer 30A 2170-2180 (1994).

25. Guan, X. Cancer metastases: challenges and opportunities. Acta Pharm. Sin. B 5, 402-418 (2015).

26. Winer, A. et al. Inhibition of breast cancer metastasis by presurgical treatment with an oral matrix metalloproteinase inhibitor: a preclinical proofof-principle study. Mol. Cancer Ther. 15, 2370-2377 (2016).

27. Jones, R., Green, T. \& Elvin, P. in Cancer Metastasis: Biologic Basis and Therapeutics (eds Lyden, D. Welch, D. R. \& Psaila, B.) 600-611 (Cambridge Univ. Press, 2011)

28. Overall, C. M. \& Kleifeld, O. Towards third generation matrix metalloproteinase inhibitors for cancer therapy. Br. J. Cancer 94, 941-946 (2006).

29. Khasraw, M. et al. Cilengitide with metronomic temozolomide, procarbazine, and standard radiotherap in patients with glioblastoma and unmethylated MGMT gene promoter in ExCentric, an open-label phase II trial. J. Neuro-Oncol. 128, 163-171 (2016).

30. Mundy, G. Metastasis to the bone: causes, consequences and therapeutic opportunities. Nat. Rev. Cancer 2, 584-593 (2002).

31. Kostenuik, P. J. et al. Denosumab, a fully human monoclonal antibody to RANKL, inhibits bone resorption and increases BMD in knock-in mice that express chimeric (murine/human) RANKL. J. Bone Miner. Res. 24, 182-195 (2009).

32. Body, J. J. et al. A study of the biological receptor activator of nuclear factor-kappaB ligand inhibitor, denosumab, in patients with multiple myeloma or bone metastases from breast cancer. Clin. Cancer Res. 12, 1221-1228 (2006)

33. Lipton, A. et al. Randomized active-controlled phase II study of denosumab efficacy and safety in patients with breast cancer-related bone metastases. J. Clin. Oncol. 25, 4431-4437 (2007).

34. Fizazi, K. et al. Denosumab versus zoledronic acid for treatment of bone metastases in men with castration-resistant prostate cancer: a randomised, double-blind study, Lancet 377, 813-822 (2011).

35. Stopeck, A. T. et al. Denosumab compared with zoledronic acid for the treatment of bone metastases in patients with advanced breast cancer: a randomized, double-blind study. J. Clin. Oncol. 28, 5132-5139 (2010)

36. Smith, M. R. et al. Denosumab and bone-metastasisfree survival in men with castration-resistant prostate cancer: results of a phase 3 , randomised, placebocontrolled trial. Lancet 379, 39-46 (2012).

37. Gnant, M. et al. Adjuvant denosumab in early breast cancer: disease-free survival analysis of 3,425 postmenopausal patients in the ABCSG-18 trial. J. Clin. Oncol. 36, 500-500 (2018).

38. Coleman, R. E. et al. Adjuvant denosumab in early breast cancer: first results from the international multicenter randomized phase III placebo controlled D-CARE study. J. Clin. Oncol. 36, 501-501 (2018).

39. Clezardin, P., Benzaid, I. \& Croucher, P. I. Bisphosphonates in preclinical bone oncology. Bone 49, 66-70 (2011).

40. Holen, I. \& Coleman, R. E. Anti-tumour activity of bisphosphonates in preclinical models of breast cancer. Breast Cancer Res. 12, 214 (2010).

41. Early Breast Cancer Trialists' Collaborative Group (EBCTCG). Adjuvant bisphosphonate treatment in early breast cancer: meta-analyses of individual patient data from randomised trials. Lancet 386 , 1353-1361 (2015).

42. Hu, G. H. et al. MTDH activation by $8 q 22$ genomic gain promotes chemoresistance and metastasis of poor-prognosis breast cancer. Cancer Cell 15, 9-20 (2009).

43. Steeg, P. S. et al. Evidence for a novel gene associated with low tumor metastatic potential. J. Natl. Cancer Inst. 80, 200-204 (1988).

44. Bidwell, B. N. et al. Silencing of Irf7 pathways in breast cancer cells promotes bone metastasis through immune escape. Nat. Med. 18, 1224-1231 (2012).

45. Eckhardt, B. L. et al. Genomic analysis of a spontaneous model of breast cancer metastasis to bone reveals a role for the extracellular matrix. Mol. Cancer Res. 3, 1-13 (2005).

46. Kang, Y. et al. A multigenic program mediating breast cancer metastasis to bone. Cancer Cell 3, 537-549 (2003).

47. Gumireddy, K. et al. In vivo selection for metastasis promoting genes in the mouse. Proc. Natl Acad. Sci. USA 104, 6696-6701 (2007).

48. Gobeil, S., Zhu, X. C., Doillon, C. J \& Green, M. R. A genome-wide shRNA screen identifies GAS1 as a novel melanoma metastasis suppressor gene. Genes Dev. 22, 2932-2940 (2008).

49. Moriarity, B. S. et al. A Sleeping Beauty forward genetic screen identifies new genes and pathways driving osteosarcoma development and metastasis. Nat. Genet. 47, 615-624 (2015).

50. Mudduluru, G. et al. Repositioning of drugs for intervention in tumor progression and metastasis: 
old drugs for new targets. Drug Resist. Updat. 26 10-27 (2016).

51. Gengenbacher, N., Singhal, M. \& Augustin, H. G Preclinical mouse solid tumour models: status quo, challenges and perspectives. Nat. Rev. Cancer 17, 751-765 (2017).

52. Eckhardt, B. L., Francis, P. A., Parker, B. S. \& Anderson, R. L. Strategies for the discovery and development of therapies for metastatic breast cancer. Nat. Rev. Drug Discov. 11, 479-497 (2012).

53. Tsai, J. H., Donaher, J. L., Murphy, D. A., Chau, S. \& Yang, J. Spatiotemporal regulation of epithelialmesenchymal transition is essential for squamous cell carcinoma metastasis. Cancer Cell 22, 725-736 (2012).

54. Trevino, J. G. et al. Inhibition of SRC expression and activity inhibits tumor progression and metastasis of human pancreatic adenocarcinoma cells in an orthotopic nude mouse model. Am. J. Pathol. 168, 962-972 (2006)

55. Yakes, F. M. et al. Cabozantinib (XL184), a novel MET and VEGFR2 inhibitor, simultaneously suppresses metastasis, angiogenesis, and tumor growth. Mol. Cancer Ther. 10, 2298-2308 (2011).

56. Guy, C. T. et al. Expression of the neu protooncogene in the mammary epithelium of transgenic mice induces metastatic disease. Proc. Natl Acad. Sci. USA 89 10578-10582 (1992).

57. Pang, H. et al. Differential enhancement of breast cancer cell motility and metastasis by helical and kinase domain mutations of class IA PI3K. Cancer Res. 69, 8868-8876 (2009).

58. Joyce, J. A. \& Pollard, J. W. Microenvironmental regulation of metastasis. Nat. Rev. Cancer $\mathbf{9}$, 239-252 (2009)

59. Slaney, C. Y., Rautela, J. \& Parker, B. S. The emerging role of immunosurveillance in dictating metastatic spread in breast cancer. Cancer Res. 73, 5852-5857 (2013).

60. Ottewell, P. D. et al. Castration-induced bone loss triggers growth of disseminated prostate cancer cells in bone. Endocr. Relat. Cancer 21, 769-781 (2014).

61. Sanmamed, M. F., Chester, C., Melero, I. \& Kohrt, H Defining the optimal murine models to investigate immune checkpoint blockers and their combination with other immunotherapies. Ann. Oncol. 27 1190-1198 (2016)

62. Simmons, J. K. et al. Animal models of bone metastasis. Vet. Pathol. 52, 827-841 (2015).

63. Brown, H. K., Schiavone, K., Tazzyman, S., Heymann, D. \& Chico, T. J. Zebrafish xenograft models of cancer and metastasis for drug discovery. Expert Opin. Drug Discov. 12, 379-389 (2017).

64. Enomoto, M., Siow, C. \& Igaki, T. Drosophila as a cancer model. Adv. Exp. Med. Biol. 1076, 173-194 (2018)

65. Maacha, S. \& Saule, S. Evaluation of tumor cell invasiveness in vivo: the chick chorioallantoic membrane assay. Methods Mol. Biol. 1749, 71-77 (2018).

66. Gopinathan, A., Morton, J. P., Jodrell, D. I. \& Sansom, O. J. GEMMs as preclinical models for testing pancreatic cancer therapies. Dis. Model. Mech. 8 1185-1200 (2015)

67. de Mingo Pulido, A. \& Ruffell, B. Immune regulation of the metastatic process: implications for therapy. Adv. Cancer Res. 132, 139-163 (2016).

68. Lehmann, B. D. et al. Identification of human triple-negative breast cancer subtypes and preclinical models for selection of targeted therapies. J. Clin. Invest 121, 2750-2767 (2011)

69. Tentler, J. J. et al. Patient-derived tumour xenografts as models for oncology drug development. Nat. Rev. Clin. Oncol. 9, 338-350 (2012).

70. DeRose, Y. S. et al. Tumor grafts derived from women with breast cancer authentically reflect tumor pathology, growth, metastasis and disease outcomes. Nat. Med. 17, 1514-1520 (2011).

71. Lallo, A., Schenk, M. W., Frese, K. K., Blackhall, F. \& Dive, C. Circulating tumor cells and CDX models as a tool for preclinical drug development. Transl Lung Cancer Res. 6, 397-408 (2017).

72. Odenthal, J., Takes, R. \& Friedl, P. Plasticity of tumor cell invasion: governance by growth factors and cytokines. Carcinogenesis 37, 1117-1128 (2016)

73. Erler, J. T. et al. Hypoxia-induced lysyl oxidase is a critical mediator of bone marrow cell recruitment to form the premetastatic niche. Cancer Cell 15, 35-44 (2009).

74. Zinonos, I. et al. Pharmacologic inhibition of bone resorption prevents cancer-induced osteolysis but enhances soft tissue metastasis in a mouse model of osteolytic breast cancer. Int. J. Oncol. 45, 532-540 (2014).

75. Harper, K. L. et al. Mechanism of early dissemination and metastasis in Her2(+) mammary cancer. Nature 540, 588-592 (2016)

76. Husemann, Y. et al. Systemic spread is an early step in breast cancer. Cancer Cell 13, 58-68 (2008).

77. Quayle, L., Ottewell, P. D. \& Holen, I. Bone metastasis: molecular mechanisms implicated in tumour cell dormancy in breast and prostate cancer. Curr. Cancer Drug Targets 15, 469-480 (2015).

78. Romero, I., Garrido, F. \& Garcia-Lora, A. M. Metastases in immune-mediated dormancy: a new opportunity for targeting cancer. Cancer Res. 74, 6750-6757 (2014).

79. van der Toom, E. E., Verdone, J. E. \& Pienta, K. J. Disseminated tumor cells and dormancy in prostate cancer metastasis. Curr. Opin. Biotechnol. 40, 9-15 (2016).

80. Zhang, X. H. F., Giuliano, M., Trivedi, M. V., Schiff, R. \& Osborne, C. K. Metastasis dormancy in estrogen receptor-positive breast cancer. Clin. Cancer Res. 19 6389-6397 (2013).

81. Morris, V. L. et al. Mammary carcinoma cell lines of high and low metastatic potential differ not in extravasation but in subsequent migration and growth. Clin. Exp. Metastasis 12, 357-367 (1994).

82. Krall, J. A. et al. The systemic response to surgery triggers the outgrowth of distant immune-controlled tumors in mouse models of dormancy. Sci. Transl Med. 10, eaan3464 (2018)

83. Bragado, P. et al. TGF- $\beta 2$ dictates disseminated tumour cell fate in target organs through TGF- $\beta$-RIII and $\mathrm{p} 38 \alpha / \beta$ signalling. Nat. Cell Biol. 15, 1351-1361 (2013).

84. Fischer, K. R. et al. Epithelial-to-mesenchymal transition is not required for lung metastasis but contributes to chemoresistance. Nature $\mathbf{5 2 7}$ 472-476 (2015)

85. Zheng, X. et al. Epithelial-to-mesenchymal transition is dispensable for metastasis but induces chemoresistance in pancreatic cancer. Nature 527 525-530 (2015)

86. Gong, C. et al. Potentiated DNA damage response in circulating breast tumor cells confers resistance to chemotherapy. J. Biol. Chem. 290, 14811-14825 (2015).

87. Ottewell, P. D. et al. Zoledronic acid has differential antitumor activity in the pre- and postmenopausal bone microenvironment in vivo. Clin. Cancer Res. 20, 2922-2932 (2014).

88. Coleman, R. E. et al. Breast-cancer adjuvant therapy with zoledronic acid. N. Engl. J. Med. 365, 1396-1405 (2011)

89. Lowes, L. E. et al. Circulating tumor cells (CTC) and cell-free DNA (cfDNA) workshop 2016: scientific opportunities and logistics for cancer clinical trial incorporation. Int. J. Mol. Sci. 17, E1505 (2016).

90. Christie, E. L., Dawson, S. J. \& Bowtell, D. D. Blood worth bottling: circulating tumor DNA as a cancer biomarker. Cancer Res. 76, 5590-5591 (2016).

91. Gold, B., Cankovic, M., Furtado, L. V., Meier, F. \& Gocke, C. D. Do circulating tumor cells, exosomes, and circulating tumor nucleic acids have clinical utility? a report of the association for molecular pathology. J. Mol. Diagn. 17, 209-224 (2015).

92. Xu, R. et al. Extracellular vesicles in cancer implications for future improvements in cancer care. Nat. Rev. Clin. Oncol. 15, 617-638 (2018).

93. Cabel, L. et al. Clinical potential of circulating tumour DNA in patients receiving anticancer immunotherapy. Nat. Rev. Clin. Oncol. 15, 639-650 (2018).

94. Anfossi, S., Babayan, A., Pantel, K. \& Calin, G. A. Clinical utility of circulating non-coding RNAs - an update. Nat. Rev. Clin. Oncol. 15, 541-563 (2018).

95. Chatterjee, A., Rodger, E. J. \& Eccles, M. R. Epigenetic drivers of tumourigenesis and cancer metastasis. Semin. Cancer Biol. 51, 149-159 (2018).

96. Simon, R. M., Paik, S. \& Hayes, D. F. Use of archived specimens in evaluation of prognostic and predictiv biomarkers. J. Natl Cancer Inst. 101, 1446-1452 (2009).

97. Steeg, P. S., Camphausen, K. A. \& Smith, Q. R. Brain metastases as preventive and therapeutic targets. Nat. Rev. Cancer 11, 352-363 (2011).

98. Lockman, P. R. et al. Heterogeneous blood-tumor barrier permeability determines drug efficacy in experimental brain metastases of breast cancer. Clin. Cancer Res. 16, 5664-5678 (2010).

99. Osswald, M. et al. Impact of blood-brain barrier integrity on tumor growth and therapy response in brain metastases. Clin. Cancer Res. 22, 6078-6087 (2016).
100. Lester, R. M. \& Olbertz, J. Early drug development: assessment of proarrhythmic risk and cardiovascular safety. Expert Rev. Clin. Pharmacol. 9, 1611-1618 (2016).

101. Loehrer, P. J. Sr. et al. A randomized comparison of cisplatin alone or in combination with methotrexate, vinblastine, and doxorubicin in patients with metastatic urothelial carcinoma: a cooperative group study. J. Clin. Oncol. 10, 1066-1073 (1992)

102. Grossman, H. B. et al. Neoadjuvant chemotherapy plus cystectomy compared with cystectomy alone for locally advanced bladder cancer. N. Engl. J. Med. 349 , 859-866 (2003).

103. Early Breast Cancer Trialists' Collaborative Group. Tamoxifen for early breast cancer: an overview of the randomised trials. Lancet 351, 1451-1467 (1998).

104. Pantel, K. \& Otte, M. Occult micrometastasis: enrichment, identification and characterization of single disseminated tumour cells. Semin. Cancer Biol. 11, 327-337 (2001).

105. Smith, M. R. et al. Natural history of rising serum prostate-specific antigen in men with castrate nonmetastatic prostate cancer. J. Clin. Oncol. 23 2918-2925 (2005).

106. Abbosh, C., Birkbak, N. J. \& Swanton, C. Early stage NSCLC - challenges to implementing ctDNA-based screening and MRD detection. Nat. Rev. Clin. Oncol. 15, 577-586 (2018)

107. Alix-Panabieres, C. \& Pantel, K. Clinical applications of circulating tumor cells and circulating tumor DNA as liquid biopsy. Cancer Discov. 6, 479-491 (2016).

108. Siva, S., MacManus, M. P., Martin, R. F. \& Martin, O. A. Abscopal effects of radiation therapy: a clinical review for the radiobiologist. Cancer Lett. 356, 82-90 (2015).

109. Demaria, S., Coleman, C. N. \& Formenti, S. C. Radiotherapy: changing the game in immunotherapy. Trends Cancer 2, 286-294 (2016).

110. Weichselbaum, R. R., Liang, H., Deng, L. \& Fu, Y. X Radiotherapy and immunotherapy: a beneficial liaison? Nat. Rev. Clin. Oncol. 14, 365-379 (2017).

111. Bellmunt, J. et al. Bladder cancer: ESMO Practice Guidelines for diagnosis, treatment and follow-up. Ann. Oncol. 25, (Suppl. 3), iii40-iii48 (2014).

112. American Cancer Society. Treatment of ductal carcinoma in situ (DCIS). American Cancer Society https://www.cancer.org/cancer/breast-cancer/ treatment/treatment-of-breast-cancer-by-stage/ treatment-of-ductal-carcinoma-in-situ-dcis (2018).

113. Paradiso, A. \& Formenti, S. Hereditary breast cancer clinical features and risk reduction strategies. Ann. Oncol. 22, i31-i36 (2011).

114. Smith, W. \& Saba, N. Retinoids as chemoprevention for head and neck cancer: where do we go from here? Crit. Rev. Oncol. Hematol. 55, 143-152 (2005).

115. Sylvester, R. J., van der Meijden, A. P. M. \& Lamm, D. L Intravesical bacillus Calmette-Guerin reduces the risk of progression in patients with superficial bladder cancer: a meta-analysis of the published results of randomized clinical trials. J. Urol. 168, 1964-1970 (2002).

116. Steinbach, G. et al. The effect of celecoxib, a cyclooxygenase- 2 inhibitor, in familial adenomatous polyposis. N. Engl. J. Med. 342, 1946-1952 (2000)

117. U.S. Food and Drug Administration. FDA approves apalutamide for non-metastatic castration-resistant prostate cancer. FDA https://www.fda.gov/Drugs/ InformationOnDrugs/ApprovedDrugs/ucm596796. htm (2018).

118. Higano, C. S. Does nonmetastatic castration-resistant prostate cancer still exist? Nat. Rev. Clin. Oncol. 15 , 350-351 (2018).

119. Morone, M. et al. Whole-body MRI: current applications in oncology. Am. J. Roentgenol 209 W336-W349 (2017)

120. Han, S., Woo, S., Kim, Y. J. \& Suh, C. H. Impact of (68) Ga-PSMA PET on the management of patients with prostate cancer: a systematic review and metaanalysis. Eur. Urol. 74, 179-190 (2018).

121. Steeg, P. S. Perspective: the right trials. Nature $\mathbf{4 8 5}$, S58-S59 (2012)

122. Abbosh, C. et al. Phylogenetic ctDNA analysis depicts early-stage lung cancer evolution. Nature $\mathbf{5 4 5}$ 446-451 (2017)

123. Beaver, J. A. et al. Detection of cancer DNA in plasma of patients with early-stage breast cancer. Clin. Cancer Res. 20, 2643-2650 (2014).

124. Garcia-Murillas, I. et al. Mutation tracking in circulating tumor DNA predicts relapse in early breast cancer. Sci. Transl Med. 7, 302ra133 (2015)

125. Kim, C. \& Prasad, V. Strength of validation for surrogate end points used in the US Food and Drug 
Administration's approval of oncology drugs. Mayo Clin. Proc. 91, 713-725 (2016)

126. Shaw, J. A. et al. Genomic analysis of circulating cell-free DNA infers breast cancer dormancy. Genome Res. 22, 220-231 (2012).

127. Leibovich, B. C. et al. Prediction of progression after radical nephrectomy for patients with clear cell renal cell carcinoma: a stratification tool for prospective clinical trials. Cancer 97, 1663-1671 (2003).

128. Dowsett, M. et al. Prediction of risk of distant recurrence using the 21-gene recurrence score in node-negative and node-positive postmenopausal patients with breast cancer treated with anastrozole or tamoxifen: a TransATAC study. J. Clin. Oncol. 28, 1829-1834 (2010)

129. Zhang, J. et al. Hypo- or conventionally fractionated radiotherapy combined with chemotherapy in patients with limited stage small cell lung cancer. Radi. Oncol. 12, 51 (2017)

130. Peixoto, R. D. et al. Prognostic factors and sites of metastasis in unresectable locally advanced pancreatic cancer. Cancer Med 4, 1171-1177 (2015).

131. Hallet, J. et al. Factors influencing recurrence following initial hepatectomy for colorectal liver metastases. Br. J. Surg. 103, 1366-1376 (2016).

132. U.S. Food and Drug Administration. Hematology/ oncology (cancer) approvals \& safety notifications. FDA http://wayback.archive-it.org/7993/ 20170111064250/http://www.fda.gov/Drugs InformationOnDrugs/ApprovedDrugs/ucm279174. htm (2016).

133. Furlow, B. US FDA advisory panel rejects early denosumab therapy. Lancet Oncol. 13, e94 (2012).

134. U.S. Food and Drug Administration. Guidance for industry: clinical trial endpoints for the approval of cancer drugs and biologics. FDA https://www.fda.gov/ downloads/drugsGuidanceCompliance RegulatoyInformation/Guidance/UCM071590.pdf (2007).

135. U.S. Food and Drug Administration. Biomarker guidances and reference materials. FDA https:/ www.fda.gov/Drugs/DevelopmentApprovalProcess/ DrugDevelopmentToolsQualificationProgram/ BiomarkerQualificationProgram/ucm536018.htm (2018).

136. Hammond, M. E. \& Taube, S. E. Issues and barriers to development of clinically useful tumor markers: a development pathway proposal. Semin. Oncol. 29 213-221 (2002)

137. Olsson, E. et al. Serial monitoring of circulating tumor DNA in patients with primary breast cancer for detection of occult metastatic disease. $E M B O \mathrm{Mol}$. Med. 7, 1034-1047 (2015)

138. Logan, A. C. et al. Immunoglobulin and T cell receptor gene high-throughput sequencing quantifies minimal residual disease in acute lymphoblastic leukemia and predicts post-transplantation relapse and survival. Biol. Blood Marrow Transplant 20, 1307-1313 (2014).

139. U.S. Food and Drug Administration. FDA granted accelerated approval to blinatumomab (Blincyto, Amgen Inc.) for the treatment of adult and pediatric patients with $B$ cell precursor acute lymphoblastic leukemia. FDA https://www.fda.gov/drugs/ informationondrugs/approveddrugs/ucm603171.htm (2018).

140. Young, G. P. et al. A cross-sectional study comparing a blood test for methylated BCAT1 and IKZF1 tumorderived DNA with CEA for detection of recurrent colorectal cancer. Cancer Med. 5, 2763-2772 (2016).

141. Fleming, T. R. \& Powers, J. H. Biomarkers and surrogate endpoints in clinical trials. Statist. Med. 31 2973-2984 (2012).

142. U.S. Food and Drug Administration. Principles for codevelopment of an in vitro companion diagnostic device with a therapeutic product. FDA https://www.fda.gov/downloads/MedicalDevices/ DeviceRegulationandGuidance/GuidanceDocuments/ UCM510824.pdf (2016).

143. European Medicines Agency. Reflection paper for laboratories that perform the analysis or evaluation of clinical trial samples. EMA https://www.ema.europa. eu/documents/regulatory-procedural-guideline/ reflection-paper-laboratories-perform-analysisreflection-paper-laboratories-perform-analysis-
evaluation-clinical-trial-samples en.pdf (2012).

144. Nikshoar, M. S. et al. Metas-Chip precisely identifies presence of micrometastasis in live biopsy samples by label free approach. Nat. Commun. 8, 2175 (2017).

145. Xiong, H. et al. High-contrast fluorescence detection of metastatic breast cancer including bone and liver micrometastases via size-controlled $\mathrm{pH}$-activatable water-soluble probes. Adv. Mater. 29, 1700131 (2017).
146. Neuschmelting, V., Lockau, H., Ntziachristos, V. Grimm, J. \& Kircher, M. F. Lymph node micrometastases and in-transit metastases from melanoma: in vivo detection with multispectral optoacoustic imaging in a mouse model. Radiology 280, 137-150 (2016).

147. Zevon, M. et al. CXCR-4 targeted, short wave infrared (SWIR) emitting nanoprobes for enhanced deep tissue imaging and micrometastatic cancer lesion detection. Small 11, 6347-6357 (2015).

148. Zhou, Z. et al. MRI detection of breast cancer micrometastases with a fibronectin-targeting contrast agent. Nat. Commun. 6, 7984 (2015).

149. Garrido-Laguna, I. et al. Tumor engraftment in nude mice and enrichment in stroma- related gene pathways predict poor survival and resistance to gemcitabine in patients with pancreatic cancer. Clin. Cancer Res. 17, 5793-5800 (2011).

150. Sledge, G. W. et al. Effect of matrix metalloproteinase inhibitor batimastat on breast cancer regrowth and metastasis in athymic mice. J. Natl Cancer Inst. 87, 1546-1550 (1995).

151. Maekawa, K., Sato, H., Furukawa, M. \& Yoshizaki, T. Inhibition of cervical lymph node metastasis by marimastat (BB-2516) in an orthotopic oral squamous cell carcinoma implantation model. Clin. Exp. Metastasis 19, 513-518 (2002)

152. Gaggioli, C. et al. Fibroblast-led collective invasion of carcinoma cells with differing roles for RhoGTPases in leading and following cells. Nat. Cell Biol. 9, 1392-1400 (2007).

153. Olive, K. P. et al. Inhibition of Hedgehog signaling enhances delivery of chemotherapy in a mouse model of pancreatic cancer. Science 324, 1457-1461 (2009).

154. Froeling, F. E. et al. Retinoic acid-induced pancreatic stellate cell quiescence reduces paracrine Wnt-betacatenin signaling to slow tumor progression. Gastroenterology 141, 1486-1497 (2011).

155. Carapuca, E. F. et al. Anti-stromal treatment together with chemotherapy targets multiple signalling pathways in pancreatic adenocarcinoma. J. Pathol. 239, 286-296 (2016).

156. Sandler, A. et al. Paclitaxel-carboplatin alone or with bevacizumab for non-small-cell lung cancer. N. Engl. J. Med. 355, 2542-2550 (2006).

157. Hurwitz, $\mathrm{H}$. et al. Bevacizumab plus irinotecan fluorouracil, and leucovorin for metastatic colorectal cancer. N. Engl. J. Med. 350, 2335-2342 (2004).

158. Harney, A. S. et al. Real-time imaging reveals local, transient vascular permeability, and tumor cell intravasation stimulated by TIE2hi macrophagederived VEGFA. Cancer Discov. 5, 932-943 (2015).

159. Noy, R. \& Pollard, J. W. Tumor-associated macrophages: from mechanisms to therapy. Immunity 41, 49-61 (2014)

160. Chiang, S. P., Cabrera, R. M. \& Segall, J. E. Tumor cell intravasation. Am. J. Physiol. Cell Physiol. 311, C1-C14 (2016).

161. Gupta, G. P. \& Massague, J. Platelets and metastasis revisited: a novel fatty link. J. Clin. Invest. 114 1691-1693 (2004).

162. Labelle, M., Begum, S. \& Hynes, R. O. Platelets guide the formation of early metastatic niches. Proc. Natl Acad. Sci. USA 111, E3053-E3061 (2014).

163. Uppal, A., Wightman, S. C., Ganai, S. Weichselbaum, R. R. \& An, G. Investigation of the essential role of platelet-tumor cell interactions in metastasis progression using an agent-based model. Theor. Biol. Med. Model. 11, 17 (2014).

164. Elwood, P. C. et al. Aspirin in the treatment of cancer: reductions in metastatic spread and in mortality: a systematic review and meta-analyses of published studies. PLOS ONE 11, e0152402 (2016).

165. Hoshino, A. et al. Tumour exosome integrins determine organotropic metastasis. Nature 527 329-335 (2015)

166. Melo, S. A. et al. Glypican-1 identifies cancer exosomes and detects early pancreatic cancer. Nature 523, 177-182 (2015).

167. Swierczak, A. et al. The promotion of breast cancer metastasis caused by inhibition of CSF-1 R/CSF-1 signaling is blocked by targeting the G-CSF receptor Cancer Immunol. Res. 2, 765-776 (2014).

168. Wculek, S. K. \& Malanchi, I. Neutrophils support lung colonization of metastasis-initiating breast cancer cells. Nature 528, 413-417 (2015)

169. Cao, Y. et al. BMP4 inhibits breast cancer metastasis by blocking myeloid-derived suppressor cell activity. Cancer Res. 74, 5091-5102 (2014).
170. Klein, C. A. Framework models of tumor dormancy from patient-derived observations. Curr. Opin. Genet Dev. 21, 42-49 (2011)

171. Lindemann, F., Schlimok, G., Dirschedl, P., Witte, J. $\&$ Riethmuller, G. Prognostic significance of micrometastatic tumour cells in bone marrow of colorectal cancer patients. Lancet 340, 685-689 (1992).

172. Mansi, J. L. et al. Bone marrow micrometastases in primary breast cancer: prognostic significance after 6 years' follow-up. Eur. J. Cancer 27, 1552-1555 (1991).

173. Pantel, K. et al. Frequency and prognostic significance of isolated tumour cells in bone marrow of patients with non-small-cell lung cancer without overt metastases. Lancet 347, 649-653 (1996).

174. Aguirre-Ghiso, J. A. Models, mechanisms and clinical evidence for cancer dormancy. Nat. Rev. Cancer 7, 834-846 (2007)

175. Shiozawa, Y. et al. Human prostate cancer metastases target the hematopoietic stem cell niche to establish footholds in mouse bone marrow. J. Clin. Invest. 121, 1298-1312 (2011).

176. Schepers, K. et al. Myeloproliferative neoplasia remodels the endosteal bone marrow niche into a self-reinforcing leukemic niche. Cell Stem Cell 13 285-299 (2013).

177. Zhao, L. et al. Recruitment of a myeloid cell subset (CD11b/Gr1 (mid)) via CCL2/CCR2 promotes the development of colorectal cancer liver metastasis. Hepatology 57, 829-839 (2013).

178. Dai, J. L. et al. Bone morphogenetic protein-6 promotes osteoblastic prostate cancer bone metastases through a dual mechanism. Cancer Res. 65, 8274-8285 (2005).

179. Huang, D. C. et al. Parathyroid hormone-related protein: potential therapeutic target for melanoma invasion and metastasis. Endocrinology 155, 3739-3749 (2014).

180. Tanaka, H. et al. Monoclonal antibody targeting of $\mathrm{N}$-cadherin inhibits prostate cancer growth, metastasis and castration resistance. Nat. Med. 16, 1414-1420 (2010).

181. Overdevest, J. B. et al. CD24 offers a therapeutic target for control of bladder cancer metastasis based on a requirement for lung colonization. Cancer Res. 71, 3802-3811 (2011).

182. Casar, B. et al. Blocking of CDCP1 cleavage in vivo prevents Akt-dependent survival and inhibits metastatic colonization through PARP1-mediated apoptosis of cancer cells. Oncogene 31, 3924-3938 (2012).

183. Park, C. S. et al. Therapeutic targeting of tetraspanin8 in epithelial ovarian cancer invasion and metastasis. Oncogene 35, 4540-4548 (2016)

184. Remacle, A. G. et al. Selective function-blocking monoclonal human antibody highlights the important role of membrane type- 1 matrix metalloproteinase (MT1-MMP) in metastasis. Oncotarget 8, 2781-2799 (2017).

185. Kitamura, T. et al. Inactivation of chemokine (C-C motif) receptor 1 (CCR1) suppresses colon cancer liver metastasis by blocking accumulation of immature myeloid cells in a mouse model. Proc. Natl Acad. Sci. USA 107, 13063-13068 (2010).

186. Mohammad, K. S. et al. TGF-beta-RI kinase inhibitor SD-208 reduces the development and progression of melanoma bone metastases. Cancer Res. $\mathbf{7 1}$, 175-184 (2011)

187. Sato, M. et al. Differential proteome analysis identifies TGF-beta-related pro-metastatic proteins in a 4T1 murine breast cancer model. PLOS ONE 10 e0126483 (2015).

188. Sadok, A. et al. Rho kinase inhibitors block melanoma cell migration and inhibit metastasis. Cancer Res. 75 , 2272-2284 (2015).

189. Said, N., Smith, S., Sanchez-Carbayo, M. \& Theodorescu, D. Tumor endothelin-1 enhances metastatic colonization of the lung in mouse xenograft models of bladder cancer. J. Clin. Invest. 121, 132-147 (2011).

190. Bondareva, A. et al. The lysyl oxidase inhibitor beta-aminopropionitrile, diminishes the metastatic colonization potential of circulating breast cancer cells. PLOS ONE 4, e5620 (2009).

191. Tang, H. et al. Lysyl oxidase drives tumour progression by trapping EGF receptors at the cell surface. Nat. Commun. 8, 14909 (2017)

192. Withana, N. P. et al. Cathepsin B inhibition limits bone metastasis in breast cancer. Cancer Res. 72, 1199-1209 (2012). 
193. Li, Y. Z. et al. Suppression of cancer relapse and metastasis by inhibiting cancer stemness. Proc. Nat Acad. Sci. USA 112, 1839-1844 (2015).

194. Saini, U. et al. Elevated STAT3 expression in ovarian cancer ascites promotes invasion and metastasis: a potential therapeutic target. Oncogene 36 168-181 (2017)

195. Wee, Z. N. et al. IRAK 1 is a therapeutic target that drives breast cancer metastasis and resistance to paclitaxel. Nat. Commun. 6, 8746 (2015).

196. Tabaries, S. et al. Lyn modulates Claudin-2 expression and is a therapeutic target for breast cancer liver metastasis. Oncotarget 6, 9476-9487 (2015).

197. Campaner, E. et al. A covalent PIN1 inhibitor selectively targets cancer cells by a dual mechanism of action. Nat. Commun. 8, 15772 (2017).

198. Andrews, F. H. et al. Dual-activity PI3K-BRD4 inhibitor for the orthogonal inhibition of MYC to block tumor growth and metastasis. Proc. Natl Acad. Sci. USA 114 E1072-E1080 (2017).

199. Ye, T. H et al. Inhibition of Stat3 signaling pathway by nifuroxazide improves antitumor immunity and impairs colorectal carcinoma metastasis. Cell Death Dis. 8, e2534 (2017).

200. Dasgupta, A., Sawant, M. A., Kavishwar, G., Lavhale, M. \& Sitasawad, S. AECHL-1 targets breast cancer progression via inhibition of metastasis, prevention of EMT and suppression of Cancer Stem Cell characteristics. Sci. Rep. 6, 38045 (2016).

201. Haak, A. J. et al. Pharmacological inhibition of myocardin-related transcription factor pathway blocks lung metastases of RhoC-overexpressing melanoma. Mol. Cancer Ther. 16, 193-204 (2017).

202. Fan, L. C. et al. Regorafenib (Stivarga) pharmacologically targets epithelial-mesenchymal transition in colorectal cancer. Oncotarget 7 64136-64147 (2016).

203. Pencheva, N., Buss, C. G., Posada, J., Merghoub, T. \& Tavazoie, S. F. Broad-spectrum therapeutic suppression of metastatic melanoma through nuclear hormone receptor activation. Cell 156, 986-1001 (2014).

204. Cadamuro, M. et al. Low-dose paclitaxel reduces S100A4 nuclear import to inhibit invasion and hematogenous metastasis of cholangiocarcinoma. Cancer Res. 76, 4775-4784 (2016).

205. Bartholomeusz, C. et al. MEK inhibitor selumetinib (AZD6244; ARRY-142886) prevents lung metastasis in a triple-negative breast cancer xenograft model. Mol. Cancer Ther. 14, 2773-2781 (2015).

206. Huang, F. K. et al. Targeted inhibition of fascin function blocks tumour invasion and metastatic colonization. Nat. Commun. 6, 7465 (2015).
207. Miao, Y. R. et al. Inhibition of established micrometastases by targeted drug delivery via cell surface-associated GRP78. Clin. Cancer Res. 19, 2107-2116 (2013)

208. Lee, C. H. et al. Sensitization of B16 tumor cells with a CXCR4 antagonist increases the efficacy of immunotherapy for established lung metastases. Mol. Cancer Ther. 5, 2592-2599 (2006)

209. Yao, H., Veine, D. M. \& Livant, D. L. Therapeutic inhibition of breast cancer bone metastasis progression and lung colonization: breaking the vicious cycle by targeting $\alpha 5 \beta 1$ integrin. Breast Cancer Res. Treat. 157, 489-501 (2016).

210. Oian, B. J. et al. MTDH/AEG-1-based DNA vaccine suppresses lung metastasis and enhances chemosensitivity to doxorubicin in breast cancer. Cancer Immunol. Immunother. 60, 883-893 (2011).

211. Lin, M. C., Lin, Y. C., Chen, S. T., Young, T. H. \& Lou, P. J. Therapeutic vaccine targeting Epstein-Barr virus laten protein, LMP1, suppresses LMP1-expressing tumor growth and metastasis in vivo. BMC Cancer 17, 18 (2017).

212. Edward, M., Gold, J. \& Mackie, R. Retinoic acidinduced inhibition of metastatic melanoma cell lung colonization and adhesion to endothelium and subendothelial extracellular matrix. Clin. Exp. Metast. 10, 61-67 (1992).

213. Wang, N. et al. The IGF-Trap: novel inhibitor of carcinoma growth and metastasis. Mol. Cancer Ther 14, 982-993 (2015)

214. Xu, W. et al. The systemic delivery of an oncolytic adenovirus expressing decorin inhibits bone metastasis in a mouse model of human prostate cancer. Gene Ther. 22, 247-256 (2015).

215. Ernsting, M. J. et al. Targeting of metastasis promoting tumor-associated fibroblasts and modulation of pancreatic tumor-associated stroma with a carboxymethylcellulose-docetaxel nanoparticle. J. Control. Release 206, 122-130 (2015).

216. Shimojo, Y. et al. Attenuation of reactive oxygen species by antioxidants suppresses hypoxia-induced epithelial-mesenchymal transition and metastasis of pancreatic cancer cells. Clin. Exp. Metastasis 30 143-154 (2013)

217. Kang, T. et al. Nanoparticles coated with neutrophil membranes can effectively treat cancer metastasis. ACS Nano 11, 1397-1411 (2017).

\section{Acknowledgements}

The authors thank F. Balkwill, R. Berg, M. Drysdale, S. Eccles, P. Elvin, A. Harris, C. Ireson, L. Machesky, R. McLeod, R. Muschel, H. Newell, M. Pittman, B. Roman, C. Santos, N. Sibson, A. Smith and I. Waddell for their participation in the joint Cancer Research UK (CRUK) and Cancer Therapeutics CRC Australia (CTX) Metastasis Working Group that formed the basis for the generation of this manuscript. R.L.A. acknowledges support from the National Breast Cancer Foundation of Australia.

\section{Author contributions}

R.L.A., J.E., R.J., J.F.M., P.S.S., A.D.W. and J.W.A.R. researched data for article. R.L.A., R.C.C., J.E., J.A.H., D.J., P.S.J., R.J., J.F.M., J.A.S., P.S.S., M.S., W.T., A.D.W. and J.W.A.R. made substantial contributions to discussions of the content R.LA, T.B, R.C. J.E., J.H S.K., D J., PS.J, R.J., J.F.M., M.B.P., J.A.S., P.S.S., M.S., W.T., A.D.W. and J.W.A.R. contributed to the writing of the manuscript. R.L.A., T.B., J.C., R.C.C., J.E., J.A.H., S.K., P.S.J., R.J., J.F.M., J.A.S., P.S.S., M.S., A.D.W. and J.W.A.R. reviewed and/or edited the manuscript before submission.

\section{Competing interests}

J.E. has received funding for clinical trials, honoraria speaker's fees and support to attend conferences from Bristol Myers Squibb. J.A.H. owns stock in and is an employee and company director of Vivactiv Limited (consultancy). J.A.S. is a member of the Scientific Advisory Board (NGS) for Qiagen and sits on the Council of the International Journal of Experimental Pathology. P.S.S. receives research funding from Medlmmune. All authors are employees of their respective organizations and declare no other competing interests.

\section{Publisher's note}

Springer Nature remains neutral with regard to jurisdictional claims in published maps and institutional affiliations.

\section{Supplementary information}

Supplementary information is available for this paper at https://doi.org/10.1038/s41571-018-0134-8.

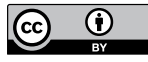

Open Access This article is licensed under a Creative Commons Attribution 4.0 International License, which permits use, sharing adaptation, distribution and reproduction in any medium or format, as long as you give appropriate credit to the original author(s) and the source, provide a link to the Creative Commons license, and indicate if changes were made. The images or other third party material in this article are included in the article's Creative Commons license, unless indicated otherwise in a credit line to the material. If material is not included in the article's Creative Commons license and your intended use is not permitted by statutory regulation or exceeds the permitted use, you will need to obtain permission directly from the copyright holder. To view a copy of this license, visit http://creativecommons.org/licenses/by/4.0/. 